Universidad de Medellín

\title{
DETERMINANTES DEL CRECIMIENTO DE LAS MICRO, PEQUEÑAS Y MEDIANAS EMPRESAS COLOMBIANAS: EL CASO DEL SECTOR METALMECÁNICO*
}

\author{
Juan Carlos Trujillo Lora** \\ Wilman Iglesias Pinedo ${ }^{* * *}$
}

Recibido: diciembre 15 de 2011 - Aceptado: diciembre 10 de 2012

\section{RESUMEN}

El crecimiento económico es una de las inquietudes centrales de la economía. Asimismo, una de las fuentes importantes del crecimiento económico en los países en vía de desarrollo es el desempeño de las micro, pequeñas y medianas empresas (MiPyME). Por su parte, el sector metalmecánico se ha perfilado como uno de los protagonistas del crecimiento industrial colombiano. De ahí que el objetivo de este artículo es estimar los determinantes del crecimiento industrial de las MiPyME del sector metalmecánico colombiano durante el cuatrienio 2002-2006. Para este propósito, se estiman tres modelos de datos de panel dinámicos para cada tamaño de empresa. Los resultados sugieren que la competencia local y los costos laborales explican en mayor magnitud el crecimiento de las MiPyME del sector.

\section{PALABRAS CLAVE}

Industrialización, tamaño de empresa, productos metálicos, modelos de datos en panel

\section{CLASIFICACIÓN JEL}

O14, L25, L61, C33

\section{CONTENIDO}

Introducción; 1. El sector metalmecánico en Colombia. 2. Revisión de la literatura. 3. Base de datos y metodología. 4. Resultados econométricos; 5. Discusión; Bibliografía; Anexos.

* Este artículo de investigación es el producto del proyecto "Caracterización y Dinámica Sectorial de las MiPyME en Colombia" vinculado al grupo de investigación Desarrollo Económico, Instituciones y Políticas Públicas para el Progreso del Caribe Colombiano (PROCARIBE) clasificado en la categoría D de COLCIENCIAS. La investigación fue financiada en su totalidad con recursos propios del grupo y su ejecución se llevó a cabo entre julio y diciembre de 2011.

** Economista, Universidad del Atlántico, Barranquilla, Colombia. Magíster en Ciencias Económicas, Universidad Nacional de Colombia, Bogotá, Colombia. Profesor del Programa de Economía, Universidad del Atlántico, Barranquilla, Colombia. Miembro activo del grupo de investigación PROCARIBE del Programa de Economía de la Universidad del Atlántico, Barranquilla, Colombia. Dirección postal: Ciudadela Universitaria, Facultad de Ciencias Económicas, Programa de Economía, Kilómetro 7, antigua vía a Puerto Colombia, Barranquilla, Colombia. Teléfono: ++ 57-5-3599472. Correo electrónico: juantrujillo@mail.uniatlantico.edu.co.

*** Economista, Universidad del Atlántico, Barranquilla, Colombia. Investigador del grupo PROCARIBE del Programa de Economía de la Universidad del Atlántico, Barranquilla, Colombia. Dirección postal: Ciudadela Universitaria, Facultad de Ciencias Económicas, Programa de Economía, Kilómetro 7, antigua vía a Puerto Colombia, Barranquilla, Colombia. Teléfono: ++ 57-5-3599472. Correo electrónico: wilmaniglesias@mail.uniatlantico.edu.co. 


\section{GROWTH DETERMINANTS IN THE MICRO, SMALL AND MEDIUM SIZE COMPANIES IN COLOMBIA: THE CASE OF THE METALLURGICAL SECTOR}

\section{ABSTRACT}

Economic growth is one of the central concerns in economics. Also, one of the major engines for economic growth in developing countries is the performance of the micro, small and medium sized enterprises (MSMEs). Meanwhile the metallurgical sector has emerged as one of the main players in the Colombian industrial growth. Hence, the objective of this paper is to estimate the determinants of industrial growth in the metallurgical sector for Colombian MSMEs for the 2002-2006. For this purpose, three panel data models are estimated for each one of the enterprise sizes. The results suggest that local competition and labor costs are the two factors that better explain the growth in the MSMEs sector.

KEY WORDS

Industrialization, company size, metal products, panel data models

\section{JEL CLASSIFICATION}

O14, L25, L61, C33

\section{CONTENT}

Introduction; 1. Metallurgical sector in Colombia. 2. Literature review. 3. Data base and methodology. 4. Econometric results; 5. Discussion; Bibliography; Attachments.

\section{DETERMINANTES DO CRESCIMENTO DAS MICRO, PEQUENAS E MEDIANAS EMPRESAS COLOMBIANAS: O CASO DO SETOR METALECÁNICO}

\section{RESUMO}

O crescimento económico é uma das inquietudes centrais da economia. Da mesma forma, uma das fontes importantes do crescimento económico nos países em via de desenvolvimento é o desempenho das micro, pequenas e medianas empresas (MiPyME). Por sua parte, o setor metalomecânico tem se perfilado como um dos protagonistas do crescimento industrial colombiano. Dai que o objetivo deste artigo é estimar os determinantes do crescimento industrial das MiPyME do setor metalomecânico colombiano durante o quatriênio 2002-2006. Para este propósito, estimam-se três modelos de dados de painel dinâmicos para cada tamanho de empresa. Os resultados sugerem que a competência local e os custos laborais explicam em maior magnitude o crescimento das MiPyME do setor.

\section{PALAVRAS CHAVE} Industrialização, tamanho da empresa, produtos metálicos, modelos de dados em painel

\section{CLASIFICAÇÃO JEL} O14, L25, L61, C33

\section{CONTEUDO}

Introdução; 1 . O setor metalomecânico na Colômbia. 2. Revisão da literatura. 3. Base de dados e metodologia. 4. Resultados econométricos; 5. Discussão; Bibliografia; Anexos. 
Determinantes del crecimiento de las micro, pequeñas y medianas empresas colombianas: el caso del sector metalmecánico

\section{INTRODUCCIÓN}

El objetivo de este artículo es analizar el crecimiento industrial de las micro, pequeñas y medianas empresas (en adelante, Mipyme) del sector metalmecánico colombiano'. Según Bell y Pavitt (1993, p. 185), este sector se ha erigido en uno de los motores del crecimiento de la industria manufacturera de varios países en vía de desarrollo. Sin embargo, en el contexto nacional, son pocos los estudios que abordan la temática del crecimiento de este sector industrial por tamaño de empresa. De hecho, la mayoría de estos trabajos se remite a aspectos descriptivos relacionados con el funcionamiento de estas empresas. Tal vez esto explique por qué diversas instituciones, tales como el Departamento Administrativo Nacional de Estadística (DANE), la Asociación Colombiana de las Micro, Pequeñas y Medianas Empresas (ACOPI) y la Fundación para la Educación Superior y el Desarrollo (FEDESARROLLO), han hecho esfuerzos durante los últimos años para obtener información estadística sobre las MiPyME en los diferentes sectores de la industria nacional.

En un contexto amplio, según Acs y Kallas (2007), la evidencia empírica ha demostrado que las Mipyme constituyen unidades de producción que impulsan y dinamizan la actividad económica de un país. De manera que se torna relevante el análisis de la contribución que hacen este tipo de empresas en el crecimiento económico. En el caso de Colombia, según el DANE, las Mipyme en 2009 representaron el 92.4\% del total de las empresas, contrataron el 48.3\% del personal ocupado, concentraron el 35.7\% de la producción bruta, el 29.7\% del valor agregado, el 23.8\% de la inversión neta y el $40.6 \%$ del consumo intermedio.

Si bien lo anterior resalta la importancia que tienen las Mipyme para potenciar el crecimiento económico del país, también es cierto que este tipo de empresas tiene una gran participación dentro de cada sector industrial de la economía. Por ello, la reflexión sobre la incidencia de variables de organización industrial, de variables externas, sociales e institucionales en el crecimiento de las MiPyME resulta esencial en el entendimiento de las heterogeneidades propias de cada tamaño de empresa en la evolución de un sector industrial determinado. Aun así, persiste un vacío respecto al análisis sobre el aporte de las Mipyme en el crecimiento por sectores industriales en Colombia.

\footnotetext{
En Colombia, de acuerdo con la definición de la ley 590 de 2000 y la ley 950 de 2004, los criterios que se emplean para clasificar a las empresas por tamaño se basa en el empleo y los activos. La Microempresa contrata hasta 10 trabajadores y cuenta con activos de menos de 501 salarios mínimos legales mensuales vigentes (smlmv). La Pequeña empresa tiene una planta de personal entre 11 y 50 trabajadores o activos entre 501 y 5,000 smlmv. La Mediana empresa cuenta con una planta de personal entre 51 y 200 trabajadores o activos totales entre 5,001 y 30,000 smlmv.
} 
La presente investigación busca llenar ese vacío. Concretamente, se pretende esclarecer la contribución de las Mipyme en el crecimiento de la producción bruta del sector metalmecánico colombiano en el cuatrienio 2002-2006. Ya se cuenta con evidencia empírica sobre la capacidad de este sector en la absorción de cambio tecnológico. Este hecho, aunado a la influencia de este sector en variables industriales clave como el empleo, el valor agregado y la inversión neta en el agregado manufacturero, amerita una indagación profunda sobre los determinantes de su crecimiento.

Este artículo está compuesto por cinco secciones, además de esta introducción. En la primera, se muestran las características generales del sector metalmecánico colombiano. La segunda sección contiene una revisión de la literatura relacionada con el análisis del crecimiento industrial. En la tercera sección se explica cómo se obtuvieron las diferentes variables que conforman la base de datos y la descripción del modelo que orienta la estimación econométrica. La cuarta sección contiene los principales resultados de la estimación. Al final se presenta la discusión de los resultados.

\section{EL SECTOR METALMECÁNICO EN COLOMBIA}

\subsection{Una mirada retrospectiva ${ }^{2}$}

El sector metalmecánico es uno de los sectores industriales de mayor dinamismo en la historia económica de Colombia. La fase embrionaria de este sector se remonta a principios del siglo XIX, en la cual se destacan una serie de propulsores que describen su evolución inicial. El primero de ellos, lo constituye la presencia de ferrerías, entre las que sobresalen La Ferrería de Pacho en Cundinamarca y las de Pradera y Amagá en Antioquia, como preludio de la industria siderúrgica del país y uno de los impulsores del proceso de industrialización nacional.

Producto del desarrollo de la industria agrícola (azucarera y cafetera) surge el segundo propulsor del sector metalmecánico colombiano. Así, comienza a despuntar la producción de maquinaria agrícola, producto terminado de las primeras ferrerías y talleres del país. Esta producción de maquinaria aun incipiente, sin embargo, generó un aumento de los ingresos locales, los que a su vez impulsaron un crecimiento sostenido de la demanda interna. La consecuencia inmediata de ese proceso fue el rápido avance tecnológico del sector, que ya se vislumbraba como protagonista del desarrollo económico nacional. Tal ímpetu sectorial coadyuvó en gran medida al desarrollo y modernización de la agricultura comercial en las postrimerías del siglo XIX.

El recuento histórico del sector metalmecánico que va desde sus inicios hasta finales de la década de los ochenta se basa exclusivamente en los documentos de Poveda (1970 y 1990). 
Determinantes del crecimiento de las micro, pequeñas y medianas empresas colombianas: el caso del sector metalmecánico

La capacitación de personal técnico se consolida como un tercer propulsor que permitió a Colombia estar a la vanguardia de la educación técnica. Es posible identificar dos vertientes en esta materia: una educación técnica-formal, impartida por la Escuela de Artes y Oficios de Medellín, y una educación en los talleres de producción impartida por técnicos extranjeros a obreros colombianos. Ya en los albores del siglo XX, el desarrollo del transporte propició el resurgimiento del sector metalmecánico del país. Desde entonces, empezó la construcción de las grandes obras de infraestructura (ferrocarriles, carreteras, etc.), el crecimiento del parque automotor y el inicio de grandes complejos fabriles metalúrgicos, localizados más que todo en Medellín, Bogotá y Cali.

Las transformaciones sociales, políticas y económicas del siglo XX, tuvieron un fuerte impacto en la evolución del sector metalmecánico. La Gran Depresión de 1929 propinó un fuerte declive en la producción manufacturera nacional, que afectó el sólido desempeño que hasta entonces había exhibido la industria metalmecánica. En el período 1935-1955 se comienza a observar la reactivación del proceso de industrialización nacional, esta vez con más vigor y enfocado a consolidar los establecimientos industriales nacientes, en especial los de la rama metalúrgica. Es así como surgen en Antioquia la primera fábrica de productos elaborados en lámina de aluminio, IMUSA, el primer taller de mecánica pesada, Talleres Centrales, y la primera fábrica de acero del país, Siderúrgica de Medellín. Más adelante, en 1947, se inaugura la Siderúrgica de Muño que acabó con el monopolio antioqueño del acero y sentaría las bases para la creación de Acerías Paz del Río en 1955. A partir de este año, Paz del Río se convierte en pieza clave para el apalancamiento de la producción del sector metalmecánico y el andamiaje del desarrollo de otras industrias.

Ahora bien, en la década de los cincuenta se observó un notable dinamismo en la creación de nuevos establecimientos industriales y en la diferenciación de los bienes como resultado de la naciente competencia por el mercado nacional. Prueba de ello es la aparición, por un lado, de muchas pequeñas y medianas empresas dedicadas a la fabricación de electrodomésticos, maquinaria agrícola y tuberías para la construcción, entre otros artefactos metálicos, y por otro, la primera iniciativa de agremiación encarnada en Acometal. A esta iniciativa le siguió la Federación Metalúrgica Colombiana (FEDEMETAL) que, desde finales de la década de 1950, representa a los industriales del sector. Entre sus metas el gremio propugna por políticas gubernamentales de desarrollo que preservaran la competitividad industrial, teniendo en cuenta la vulnerabilidad frente al manejo cambiario de la época y la minimización de los riesgos, dadas las cuantiosas inversiones de lenta maduración.

Entre el año 1960 y comienzos de 1974, la Ley 81 de 1960 estimuló mediante exenciones tributarias el crecimiento, diversificación y redistribución geográfica 
del sector metalmecánico. Al amparo de esta ley, durante el período mencionado, surgieron empresas como Hojalata y Laminados S. A. (HOLASA), creada bajo el auspicio de la Siderúrgica de Medellín en 1960; la Fábrica Colombiana de Automotores (COLMOTORES) en 1962, con una alta participación accionaria de la corporación estadounidense Chrysler; la Sociedad de Fabricación de Automotores (SOFASA) en 1969, con la participación de la multinacional francesa Renault; y la empresa de ensamblaje de aeronaves livianas Cessna. Además, y como una contribución al ánimo descentralizador de la industria de la época, se destaca la creación de nuevas plantas en ciudades intermedias tales como Barranquilla, Manizales, Pereira y Bucaramanga, entre otras. Sin embargo, en 1974, con el advenimiento de la política de liberación de las importaciones durante la administración López Michelsen, la industria en general sufrió un descenso pronunciado en la producción, ocasionando efectos nefastos que se extendieron hasta principios de los ochenta.

Como lo manifiesta Poveda (1990), solo hasta 1984 el sector metalmecánico logra alcanzar los niveles de producción bruta y valor agregado exhibidos antes de la apertura de los mercados, con lo cual se acumuló una década perdida para el sector. Desde mediados de 1983 hasta comienzos de la década de los noventa el sector metalmecánico observa una senda de crecimiento sostenida. Según Guterman (1996, p. 11), tal tendencia se refuerza en el período 1991-1994, correspondiente a la apertura económica, en el cual el crecimiento del sector metalmecánico se situó por encima del promedio industrial del país.

No obstante, esta tendencia se revierte como consecuencia de la crisis desatada a partir de 1995 con el desvanecimiento del comercio exterior industrial sumado a la revaluación que se prolonga hasta 1998. Otros factores que incidieron en la caída del sector fueron el aumento en las tasas de interés, las continuas reformas tributarias encaminadas a corregir el déficit fiscal, el rezago en la infraestructura pública regional, la inseguridad y la corrupción (Garay, 2004).

A comienzos del siglo XXI se observar nuevamente una senda de crecimiento sostenida concomitante con alzas importantes tanto en la capacidad instalada como en la inversión productiva del sector, según datos de la Encuesta de Opinión Industrial Conjunta de enero-agosto de 2004. Cabe señalar, por último, que Colombia se considera en la actualidad un importador neto de productos metalmecánicos, provenientes en su mayoría de Estados Unidos.

\subsection{Panorama actual en cifras}

De acuerdo con la información de la Encuesta Anual Manufacturera (EAM) en 2009 en Colombia existen 9,135 establecimientos dedicados a la industria. Entre ellos, 
Determinantes del crecimiento de las micro, pequeñas y medianas empresas colombianas: el caso del sector metalmecánico

1,618 -es decir, el 17.7\%-corresponden al sector metalmecánico. Este sector se compone de 13 grupos industriales (ver tabla 1) a tres dígitos de nivel de agregación teniendo en cuenta la Clasificación Industrial Internacional Uniforme (CIIU). Por otra parte, este sector aporta el $13.5 \%$ de la producción bruta de Colombia, el 11.2\% del VA, el 36.5\% de la inversión neta y contribuye con el 15\% del total de personal ocupado en el conjunto de la industria nacional.

Tabla 1. Grupos industriales del sector metalmecánico

\begin{tabular}{|c|c|}
\hline CIIU & Grupo industrial \\
\hline 271 & Industrias básicas de hierro y acero \\
\hline 272 & Industrias básicas de metales preciosos y de metales no ferrosos \\
\hline 281 & $\begin{array}{l}\text { Fabricación de productos metálicos para uso estructural, tanques, depósitos } \\
\text { y generadores de vapor }\end{array}$ \\
\hline 289 & $\begin{array}{l}\text { Fabricación de otros productos elaborados de metal y actividades de servicios } \\
\text { relacionados con el trabajo de metales }\end{array}$ \\
\hline 291 & Fabricación de maquinaria de uso general \\
\hline 292 & Fabricación de maquinaria de uso especial \\
\hline 293 & Fabricación de aparatos de uso doméstico ncp \\
\hline 341 & Fabricación de automotores y sus motores \\
\hline 342 & $\begin{array}{l}\text { Fabricación de carrocerías para vehículos automotores, fabricación de remol- } \\
\text { ques y semirremolques }\end{array}$ \\
\hline 343 & $\begin{array}{l}\text { Fabricación de partes, piezas y accesorios (autopartes) para vehículos auto- } \\
\text { motores y para sus motores }\end{array}$ \\
\hline 351 & Construcción y reparación de buques y de otras embarcaciones \\
\hline 353 & Fabricación de aeronaves y de naves espaciales \\
\hline 359 & Fabricación de otros tipos de equipo de transporte ncp \\
\hline
\end{tabular}

Fuente: elaboración propia.

\subsubsection{El caso de las Mipymes}

Una revisión de la clasificación por tamaño, localización y grupo industrial ofrece un panorama de heterogeneidad en términos del número de establecimientos y su contribución a la producción bruta y al valor agregado. Respecto a estos dos últimos indicadores de la industria metalmecánica nacional, se encuentra que las 
pequeñas y medianas empresas (pymes) predominan en cerca de cinco de los trece grupos industriales que componen dicha industria (ver tabla 2). De hecho, las pyme generan el 100\% de la producción bruta, el valor agregado y el empleo en el CIIU 351, así como más del 60\% de cada uno de estos indicadores en los CIIU 291, 292 y 343.

Las Mipymes conforman la estructura predominante del sector metalmecánico colombiano. Según la EAM, en 2009, el 94\% de los establecimientos de este sector correspondieron a las Mipymes. Además, estas empresas representaron el 55.6\% del personal ocupado, el 38.4\% de la producción bruta, el 32.3\% del valor agregado y el $23.7 \%$ de la inversión neta de todo el sector.

Tabla 2. Tamaño de la empresa de mayor representatividad en la producción bruta y el valor agregado 3

\begin{tabular}{|l|l|l|}
\hline \multirow{2}{*}{ CIIU } & \multicolumn{2}{|c|}{ Tamaño de la Empresa } \\
\cline { 2 - 3 } & \multicolumn{1}{|c|}{ Producción bruta } & \multicolumn{1}{c|}{ Valor agregado } \\
\hline 271 & Grandes & Grandes \\
\hline 272 & Pequeñas & Grandes/ Pequeñas \\
\hline 281 & Indefinido & Indefinido \\
\hline 289 & Indefinido & Indefinido \\
\hline 291 & Pymes & Pymes \\
\hline 292 & Pymes & Pymes \\
\hline 293 & Grandes & Grandes \\
\hline 341 & Grandes & Grandes \\
\hline 342 & Indefinido & Indefinido \\
\hline 343 & Medianas & Medianas \\
\hline 351 & Pymes & Pymes \\
\hline 353 & Grandes & Grandes \\
\hline 359 & Grandes & Grandes \\
\hline
\end{tabular}

Fuente: elaboración propia con base en EAM (2009).

De un total de 1,618 establecimientos del sector metalmecánico estimados en 2009, 1,521 corresponden a las Mipymes, los cuales se concentran en Bogotá y Cundinamarca con el 51\%. Le siguen Antioquia con el 18\% y Valle del Cauca con el 14\% (ver tabla 3). El resto de los Departamentos contribuye marginalmente al número de establecimientos Mipyme del sector metalmecánico.

3 En este análisis se omiten las microempresas debido a que su participación agregada tanto en la producción bruta como en el valor agregado no es representativa. 
Determinantes del crecimiento de las micro, pequeñas y medianas empresas colombianas: el caso del sector metalmecánico

El grupo industrial del sector metalmecánico que dentro de las Mipymes tiene mayor relevancia respecto al número de establecimientos, personal ocupado y valor agregado es el CIIU 289, con 23.8\%, 22.4\% y 22.3\%, respectivamente (Ver tabla 2). También se destacan en cuanto al número de establecimientos los CIIU 292 (17.4\%), 281 (16.4\%) y 291 (15.5\%). De la misma forma, estos grupos industriales contribuyen en forma significativa con el valor agregado de las Mipymes del sector. Pero es importante destacar la generación de valor agregado de las 113 Mipymes clasificadas en el grupo industrial CIIU 271, las cuales contribuyen con el 11.1\% de este indicador.

Tabla 3. Distribución de los establecimientos Mipyme del sector metalmecánico por Departamentos

\begin{tabular}{|c|c|c|c|c|c|c|c|c|c|c|c|c|c|c|c|}
\hline \multirow{2}{*}{ Departamento } & \multicolumn{15}{|c|}{ Clasificación Industrial Internacional Uniforme (CIIU) } \\
\hline & 271 & 272 & 281 & 289 & 291 & 292 & 293 & 341 & 342 & 343 & 351 & 353 & 359 & $\mathrm{TM}$ & $\%$ \\
\hline Bogotá & 45 & 14 & 107 & 169 & 114 & 114 & 16 & 5 & 30 & 68 & & 7 & 11 & 700 & 46 \\
\hline Antioquia & 17 & 12 & 43 & 82 & 42 & 40 & 4 & 4 & 7 & 12 & & & 9 & 270 & 18 \\
\hline Valle & 14 & 10 & 31 & 47 & 35 & 62 & & & 9 & 4 & & & 3 & 215 & 14 \\
\hline $\begin{array}{l}\text { Cundina- } \\
\text { marca }\end{array}$ & 5 & 5 & 18 & 9 & 11 & 10 & & 5 & 3 & 10 & & & & 76 & 5 \\
\hline Atlántico & 3 & 5 & 8 & 9 & 13 & 12 & & & 4 & 3 & 4 & & & 62 & 4 \\
\hline Santander & 3 & & 9 & 8 & 7 & 16 & & & 6 & 4 & & & & 52 & 3 \\
\hline Caldas & 4 & & 8 & 16 & 5 & 5 & & & & 3 & & & & 41 & 3 \\
\hline Otros $^{\mathrm{a}}$ & 22 & & 25 & 22 & 8 & 6 & & & 6 & 3 & 7 & & 7 & 105 & 7 \\
\hline Total & 113 & 45 & 250 & 362 & 235 & 264 & 20 & 14 & 64 & 106 & 11 & 7 & 30 & 1,521 & 100 \\
\hline
\end{tabular}

TM= Total establecimientos Mipyme del sector metalmecánico en el Departamento.

a Otros incluyen los Departamentos de Bolívar, Boyacá, Cauca, Norte de Santander, Quindío, Risaralda y Tolima.

Fuente: elaboración propia con base en EAM (2009).

Tabla 4. Participación porcentual de las principales variables industriales para las Mipyme del sector metalmecánico según CIIU

\begin{tabular}{|c|c|c|c|c|}
\hline CIIU & $\begin{array}{c}\text { Número de } \\
\text { establecimientos (\%) }\end{array}$ & $\begin{array}{c}\text { Total personal } \\
\text { ocupado (\%) }\end{array}$ & $\begin{array}{c}\text { Producción } \\
\text { bruta (\%) }\end{array}$ & $\begin{array}{c}\text { Valor } \\
\text { agregado (\%) }\end{array}$ \\
\hline 271 & 7.4 & 8.4 & 13.2 & 11.1 \\
\hline 272 & 3.0 & 3.0 & 25.6 & 5.0 \\
\hline 281 & 16.4 & 15.3 & 11.9 & 16.0 \\
\hline 289 & 23.8 & 22.4 & 16.2 & 22.3 \\
\hline 291 & 15.5 & 17.2 & 11.6 & 16.2 \\
\hline
\end{tabular}


Juan Carlos Trujillo Lora • Wilman Iglesias Pinedo

\begin{tabular}{|c|c|c|c|c|}
\hline CIIU & $\begin{array}{c}\text { Número de } \\
\text { establecimientos (\%) }\end{array}$ & $\begin{array}{c}\text { Total personal } \\
\text { ocupado (\%) }\end{array}$ & $\begin{array}{c}\text { Producción } \\
\text { bruta (\%) }\end{array}$ & $\begin{array}{c}\text { Valor } \\
\text { agregado (\%) }\end{array}$ \\
\hline 292 & 17.4 & 14.9 & 7.9 & 12.9 \\
\hline 293 & 1.3 & 1.5 & 0.6 & 0.7 \\
\hline 341 & 0.9 & 1.2 & 2.7 & 1.5 \\
\hline 342 & 4.2 & 4.5 & 3.0 & 4.1 \\
\hline 343 & 7.0 & 8.4 & 5.6 & 7.9 \\
\hline 351 & 0.7 & 1.0 & 0.8 & 1.1 \\
\hline 353 & 0.5 & 0.2 & 0.2 & 0.3 \\
\hline 359 & 2.0 & 2.0 & 0.7 & 0.8 \\
\hline
\end{tabular}

Fuente: elaboración propia con base en EAM (2009).

Cabe resaltar el desempeño sobresaliente del grupo industrial CIIU 272 en relación con la producción bruta (25.6\% del total), si se tiene en cuenta que solo está compuesto por el 3\% de los establecimientos Mipyme del sector. El resto de los 12 grupos industriales, aportan entre el 0.2\% (CIIU 353) y el 16.2\% (CIIU 289) de la producción bruta de las Mipymes del sector metalmecánico.

\section{REVISIÓN DE LA LITERATURA}

Las Mipymes han sido objeto de investigación durante los últimos años por diversas razones, aunque son pocos los estudios que abordan la explicación de su desempeño en el tiempo. La literatura sobre el crecimiento de las Mipymes se vincula a cinco ejes temáticos. En el primero de ellos se abordan los principales elementos teóricos de la teoría moderna del crecimiento económico. El segundo hace un barrido teórico de los aspectos que justifican la inclusión de la organización industrial como determinante de la evolución productiva de las Mipymes. El tercer eje resume las principales contribuciones que se han hecho respecto a la influencia de la educación y la desigualdad en el ingreso en el crecimiento económico. Un cuarto eje analiza la incidencia de la apertura comercial de un sector industrial como dinamizador de su propio crecimiento. Por último, se examina el entorno institucional de un país y sus efectos en el desarrollo de las empresas.

\subsection{Crecimiento y convergencia}

Los desarrollos contemporáneos del crecimiento económico se remiten a la teoría del crecimiento endógeno. Esta teoría hace hincapié en el papel de la inversión en capital humano, el comercio internacional y el avance tecnológico sobre la tasa 
Determinantes del crecimiento de las micro, pequeñas y medianas empresas colombianas: el caso del sector metalmecánico

de crecimiento y el proceso de convergencia de países desarrollados y en vía de desarrollo. A diferencia de los modelos de crecimiento basados en Solow (1956), la teoría del crecimiento endógeno intenta explicar cómo el progreso tecnológico se convierte en un determinante clave del crecimiento de los países. Autores como Arrow (1962) incorporan la noción de learning by doing en la explicación del crecimiento económico a largo plazo, mediante la cual el progreso tecnológico se considera un factor de producción endógeno.

A partir de la identificación de este factor, autores como Uzawa (1965), Romer (1986), Lucas (1988) y Rebelo (1991) establecen que el crecimiento es impulsado por el cambio tecnológico endógeno. En esencia, se trata de un cambio proveniente de la inversión en ciencia y tecnología y de los desbordamientos tecnológicos (spillovers) derivados de la Investigación y Desarrollo (I+D). Romer (1986) señala que el conocimiento es un insumo que muestra rendimientos crecientes debido a las externalidades positivas que genera. Bajo esta misma interpretación, Grossman y Helpman (1994) y Aghion y Howitt (1997) destacan que el capital humano procede de la acumulación formativa a través de los años de escuela y de la experticia lograda por el individuo en su lugar de trabajo. Estos autores aducen que las empresas acumulan conocimiento por medio de la I+D y este no es un bien de libre disposición, es decir, tiene un costo, por lo que este tipo de modelos se esfuerza por esclarecer cómo se difunde la tecnología entre los países. Así, el ingreso per cápita puede crecer en forma ilimitada en función del nivel de inversión en I+D, de manera que la brecha entre países desarrollados y en vía de desarrollo tenderá a expandirse. Dicho de otro modo, no se llegará a la convergencia ${ }^{4}$. En efecto, al analizar el crecimiento registrado en 113 países para el período 1960-1985, Barro (1991) encuentra que los países con menores niveles de renta per cápita en principio no exhiben una tendencia a crecer relativamente más rápido y a cerrar la brecha respecto a aquellos países con mayores niveles de renta per cápita.

\subsection{Estructura industrial}

Los estudios sobre el vínculo entre el desempeño de las empresas y la estructura industrial tienen un fuerte contenido empírico. Una de las características que identifica al sector metalmecánico es la baja concentración de las empresas, además de

\footnotetext{
4 La convergencia es un proceso por el cual regiones relativamente atrasadas crecen más rápido que aquellas que son ricas. Al respecto, cabe señalar que existen dos tipos de convergencia. La primera de ellas es la convergencia absoluta. Esta implica que, en el largo plazo, los países o regiones crecerán hasta llegar a un estado estacionario, en el cual todas las variables macroeconómicas crecen a una tasa constante. Por otro lado, se tiene la convergencia condicional, según la cual un país o región que se encuentre próximo a su estado estacionario, experimentará un descenso en su tasa de crecimiento económico. Para mayores detalles, véanse Barro y Sala-i-Martin (1992).
} 
ser uno de los sectores más competidos de la industria manufacturera (Guterman, 1996). En tal sentido, la estructura organizativa de las empresas, dado su tamaño, evidencia el grado de competitividad empresarial. Esta estructura refleja las posibilidades de crecimiento y acumulación de capital de las empresas como resultado del desempeño que tengan dentro del sector industrial al que pertenecen. La competitividad empresarial puede ser analizada a través de los salarios, la acumulación de capital y la exposición a la competencia local.

Hicks (1977) sostiene que si hay una baja en el precio de un factor productivo, su demanda debe aumentar y provoca dos posibles efectos: la contracción de la demanda de algún otro factor o el incremento de la producción. Por consiguiente, desde la perspectiva del empresario, un aumento del salario encarece el factor trabajo lo que supone que este será más reacio a contratar un mayor número de trabajadores. De ese modo, el empresario sustituye el trabajo por otro factor. El trabajador, por su parte, ante un salario más alto incrementa su productividad marginal (mayor producción), dado el incentivo de una mayor remuneración percibida por él.

Otro de los aspectos cruciales en el desarrollo del sector metalmecánico es la intensa absorción de cambio tecnológico producto de la capacidad innovadora que ofrece el sector. No obstante, la evidencia empírica muestra que, al interior del sector metalmecánico, predomina el contraste entre empresas innovadoras frente a otras que sistemáticamente imitan (Katz, 1983, p. 127). Por tal motivo, es posible considerar la tecnología como un factor variable, ya que la competencia local incentiva la adopción de nuevas tecnologías que aceleran el crecimiento industrial. Porter (1991) afirma que la competencia local, asociada al grado de concentración de empresas (clustering), acelera los procesos de imitación e innovación tecnológica, los cuales redundan en un mayor crecimiento económico. A pesar de ello, la competencia local puede tener consecuencias positivas o negativas en el crecimiento debido a que la innovación adopta rasgos diferentes dependiendo del contexto al cual se hace referencia (Lundvall, 1992; Freeman, 1994).

Pese a la importancia de las Mipymes en el desarrollo económico, siguen siendo escasos los estudios que vinculan la estructura industrial con el desempeño económico de estas empresas en el contexto internacional. Acs y Audretsch (1994) constituyen el estudio germinal de la incidencia de la estructura de mercado en el cambio tecnológico de las MiPyME. Según estos autores, la actividad económica de las pequeñas empresas hace una contribución esencial y diferente a la de las grandes empresas. Además, los autores encuentran evidencia de que la innovación tecnológica disminuye a medida que la concentración industrial aumenta. En Colombia, el principal referente sobre los efectos de la estructura industrial en el desempeño de las Mipymes es el estudio de Cardona y Cano (2005). Este estudio revela, por un 
Determinantes del crecimiento de las micro, pequeñas y medianas empresas colombianas: el caso del sector metalmecánico

lado, la convergencia en el crecimiento de las Mipymes para el período 1980-2000 y, por otro, efectos ambiguos en la evidencia de la teoría hicksiana de los salarios.

\subsection{Contexto social}

El cambio tecnológico está sujeto a un amplio rango de factores económicos y sociales que abarcan una diversidad de fenómenos difíciles de delimitar en un modelo específico (Archibugil y Michie, 1998). Entre los factores sociales, vale la pena mencionar el nivel de educación de la sociedad y el grado de desigualdad del ingreso. Por ejemplo, existe evidencia empírica que demuestra que el número de años de escolaridad afecta positivamente el crecimiento de un país (Nelson y Phelps, 1966; Romer, 1990). Asimismo, se ha demostrado que las sociedades menos desiguales en materia de ingreso ostentan mayores tasas de crecimiento en el largo plazo (Kuznets, 1955; Deininger y Squire, 1998; Aghion, 2002).

En términos de la inversión en educación, el crecimiento económico puede verse impactado desde dos aristas, como lo sugieren González, Guzmán y Pachón (1998, p. 9). Una de ellas es de carácter interno y consiste en el incremento de la productividad laboral por medio de un mayor nivel de capacitación o entrenamiento al interior de la empresa. La otra es generada por las externalidades que surgen de la interacción entre las instituciones privadas y/o públicas en las que debe recaer la responsabilidad de incentivar y mejorar las condiciones sociales para el logro de las innovaciones tecnológicas. ${ }^{5}$ Siguiendo esa orientación, Easterly (2002) argumenta que la inversión en educación es rentable en la medida en que exista una tasa de retorno mínima, de tal manera que el capital humano se traslada hacia donde obtenga mayor compensación por su uso.

De esta manera, el conocimiento productivo de una sociedad aborda en forma específica la intervención pública en la construcción de capacidades tecnológicas de la empresa ${ }^{6}$. En este sentido, se advierte que el conocimiento contribuye de manera complementaria en el proceso de crecimiento industrial. Sin embargo, subyace el problema de la distribución del conocimiento. Como lo anota Arrow (1994, p.16): "... it is not just the average level of knowledge that is relevant, but also its distribution". Más aún, para Cardona y Cano (2005) la desigual distribución del conocimiento imposibilita el logro de mayores niveles de desarrollo económico. Tal como lo manifiestan Cardona

5 Tales instituciones incluyen universidades, centros de pensamiento, agencias financieras, empresas privadas, entre otras, configurando lo que Lundvall (1992) denomina Sistema Nacional de Innovación.

6 Según Lall (1992) las capacidades tecnológicas en el ámbito de la empresa son aquellos factores requeridos para generar y administrar el cambio tecnológico. Entre estas cabe citar las habilidades personales, la experiencia en el trabajo, las estructuras institucionales y los eslabonamientos intra e intersectoriales.

Semestre Económico, volumen 15, №. 32, pp. 41-76 • ISSN 0120-6346, julio-diciembre de 2012, Medellín, Colombia 
y Cano (2005, p. 18): "en el proceso societario signado por la importancia del conocimiento no se pueden desconocer las diferencias estructurales que se derivan de la división internacional del trabajo y que imponen condiciones nada paritarias en el acceso a los beneficios de la cultura y del bienestar".

\subsection{Competitividad externa}

El comportamiento de las empresas en el crecimiento industrial se somete a la competencia en los mercados externos orientada por el proceso de globalización imperante en las economías. Gould y Ruffin (1995) subrayan la interacción del capital humano con el régimen comercial en la determinación del progreso económico de los países. A partir de ese vínculo, estos autores muestran que el grado de apertura o exposición a la competencia externa que tenga un país origina efectos externos del capital humano que están correlacionados con la evolución de su economía. Así, tal vez sería inadecuado analizar la competitividad externa de las empresas por tamaño de manera aislada. La evidencia apunta a que la capacidad de las empresas para entronizarse en el comercio internacional está ligada a otros factores consustanciales del crecimiento económico.

Amal y Rocha (2010) muestran que el desempeño exportador y la diversificación de los mercados de las Mipymes dependen de la habilidad de estas para relacionar la innovación con la capacidad de generar redes internacionales tanto de clientes como proveedores. Mientras mayores sean estas capacidades, mayor será la tendencia de las Mipymes a alcanzar mejores desempeños en los mercados foráneos. En esa misma dirección, Cheng y Yu (2008) encuentran que el ambiente externo puede incidir en la orientación internacional, toda vez que las Mipymes pueden responder de manera positiva a presiones institucionales o externas, conduciéndolas a adoptar estrategias riesgosas y acelerar su exposición internacional. En el caso de los países en desarrollo, Zhu, Hitt y Tihanyi (2007) señalan que el escenario institucional doméstico es fundamental para la competitividad internacional de las Mipymes en la medida en que proveen acceso tanto a recursos valiosos como al capital humano. No obstante, Amal y Rocha (2010) recomiendan investigaciones en grupos de países para identificar otros factores que pueden afectar el desempeño internacional de las Mipymes bajo diferentes ambientes culturales y arreglos institucionales.

\subsection{Entorno institucional}

Las instituciones, entendidas como las restricciones formales e informales que condicionan la interacción humana, constituyen un factor que define la estructura de incentivos de las sociedades (North, 1994). En consecuencia, es importante 
Determinantes del crecimiento de las micro, pequeñas y medianas empresas colombianas: el caso del sector metalmecánico

considerar la percepción que posean los actores sociales sobre el contexto institucional en el que interactúan. La corrupción emerge como uno de los obstáculos del proceso de crecimiento económico de un país. UNIDO y UNODC (2007) prestan especial atención a los efectos que embiste la corrupción en las pymes en el contexto internacional. Según UNIDO y UNODC (2007, p. 7), "available data strongly suggests that the smaller the firm is, the more likely it is to be affected by corruption". En ese mismo documento, se mencionan los principales factores que coadyuvan a que la corrupción entorpezca la actividad económica de las pymes. Entre ellos cabe destacar el alto grado relativo de informalidad, la estrecha relación entre la planta de personal que puede conllevar a una cultura donde la corrupción es tolerable, la visión inmediatista, los recursos financieros limitados, la carencia de poder de negociación e influencia sobre las instituciones estatales y la estructura del capital, la cual no demarca una separación formal entre accionistas, administradores y cuerpo directivo.

En América Latina la evidencia apunta a que la desconfianza en las instituciones por parte de los actores sociales, entre ellos las empresas, ha llegado a ribetes dramáticos. Zevallos (2003) sostiene que las instituciones sobre las cuales se cimientan los Estados latinoamericanos subordinan en gran medida el desempeño económico de las Mipymes. Este autor atribuye este hecho a la desconfianza de los ciudadanos en instituciones tales como la presidencia, los partidos políticos y el congreso, la cual propina efectos adversos sobre la actividad económica en general.

\section{BASE DE DATOS Y METODOLOGÍA}

En esta sección se presenta una descripción de la metodología y los datos utilizados para construir las variables que han incidido en el crecimiento industrial de las Mipymes del sector metalmecánico colombiano. El período de estudio fue seleccionado por la disponibilidad y fiabilidad de los datos requeridos para la estimación econométrica. La información utilizada proviene de la EAM referente a las Mipymes del sector metalmecánico ${ }^{7}$.

La variable CI (crecimiento industrial), generada a partir de la variación porcentual de la producción bruta por tamaño de empresa deflactada con el índice de precios al productor (IPP), se utiliza como variable explicada. Además, se incluyen cuatro tipos de variables explicativas que podrían influir en el CI de las Mipymes del sector metalmecánico durante el período de estudio: variables de organización industrial, variables externas, variables sociales y una variable institucional. Además, se incluye

$7 \quad$ La base de datos utilizada en este estudio se puede consultar en el anexo o está disponible para todos los lectores interesados, solicitándola al correo electrónico: juantrujillo@mail.uniatlantico.edu.co.

Semestre Económico, volumen 15, №. 32, pp. 41-76 • ISSN 0120-6346, julio-diciembre de 2012, Medellín, Colombia 
la variable crecimiento industrial rezagado para dar cuenta del efecto de convergencia de las empresas por tamaño en el período de estudio.

\subsection{Variable de convergencia}

La teoría del crecimiento neoclásica plantea que los países que se encuentran relativamente cerca de su estado estacionario experimentarán un descenso en su tasa de crecimiento económico. Como antes se acotó, este fenómeno se denomina convergencia condicional. Una analogía se podría establecer con el crecimiento del sector metalmecánico por tamaño de empresas. Específicamente, el comportamiento de la variable crecimiento industrial rezagado $\left(\mathrm{CI}_{t-1}\right)$ refleja este proceso de convergencia en las Mipymes del sector durante el cuatrienio estudiado.

Si el coeficiente de $\mathrm{CI}_{t-1}$ es negativo, entonces el crecimiento industrial exhibe un proceso de convergencia condicional. Esto quiere decir que las empresas, dado su tamaño, han crecido a tasas decrecientes ya que se encuentran próximas a alcanzar su estado estacionario.

\subsection{Variables de organización industrial}

Como se expresó más arriba, la industria manufacturera es uno de los motores del crecimiento económico de un país en la medida en que su desarrollo propicia, entre otros factores, spillovers a otros sectores de la economía. En tal sentido, el análisis de la organización industrial permite determinar las ventajas y desventajas en la competitividad y, en este caso particular, mostrar el desempeño de las Mipymes del sector metalmecánico en términos de su capacidad para encarar los desafíos del mercado nacional. A continuación se describe la construcción de las variables correspondientes a la organización industrial.

El salario promedio industrial (SPI), se calcula de la siguiente forma:

$$
S P I_{i j t}=\left[\frac{W_{i j t} / I P C}{L_{i j t}}\right]
$$

donde:

$\mathrm{W}_{i j t}=$ Sueldos y salarios en el grupo industrial por tamaño de empresa.

IPC = Índice de precios al consumidor, base 2002.

$\mathrm{L}_{i j t}=$ Personal ocupado en el grupo industrial por tamaño de empresa. 
Determinantes del crecimiento de las micro, pequeñas y medianas empresas colombianas: el caso del sector metalmecánico

Los subíndices $i$, $j$ y $t$ denotan el grupo industrial, el tamaño de la empresa y el tiempo, respectivamente. Un coeficiente de la variable SPI $>0$ significa que un incremento en el promedio de los salarios genera $\mathrm{CI}$ en las empresas del respectivo tamaño en el sector metalmecánico colombiano.

Por su parte, la relación capital-producto (RKP), se estima así:

$$
R K P_{i j t}=\left[\frac{K_{i j t} / I P P}{V A_{i j t} / I P P}\right]
$$

donde:

$\mathrm{K}_{i j t}=$ Activos fijos reales del grupo industrial por tamaño de empresa.

$\mathrm{VA}_{i j t}=$ Valor agregado del grupo industrial por tamaño de empresa.

Un coeficiente positivo de la variable RKP implicaría que la inversión industrial ha generado cambio tecnológico reflejado en un mayor CI. En otras palabras, el incremento marginal del capital provoca un aumento mayor en la producción bruta.

El Costo Laboral Unitario (CLU) se obtiene de la siguiente manera:

$$
C L U_{i j t}=\left[\frac{W_{i j t} / I P C}{Y_{i j t} / I P P}\right]
$$

donde:

$Y_{i j t}=$ Producción bruta del grupo industrial por tamaño de empresa.

En este caso, un coeficiente negativo de la variable CLU, desde la teoría hicksiana de los salarios, indicaría que el aumento del costo de la mano de obra requerido para producir una unidad de producto representa un menor $\mathrm{Cl}$ en el sector manufacturero por tamaño de empresa.

Por último, se tiene el Índice de Competencia Local (ICL):

$$
I C L_{i j t}=\left[\frac{N_{i j t} / L_{i j t}}{N_{j t} / L_{j t}}\right]
$$


donde:

$\mathrm{N}_{i j t}=$ Número de establecimientos del grupo industrial por tamaño de empresa.

$\mathrm{N}_{j t}=$ Número de establecimientos totales del sector metalmecánico por tamaño de empresa.

$\mathrm{L}_{j \mathrm{t}} \quad=$ Personal ocupado en el sector metalmecánico por tamaño de empresa.

Un coeficiente de ICL $>0$ revelaría que la presencia de un mayor número de establecimientos por tamaño de empresa en el sector metalmecánico genera un aumento del CI.

\subsection{Variables sociales}

Para el caso de las Mipymes del sector metalmecánico colombiano, se aplica una serie de indicadores utilizados como variables proxy para medir la incidencia del capital humano y de la desigualdad del ingreso en el crecimiento industrial. En particular, las variables son el Índice de Logro Educativo (ILE) y el Coeficiente de Gini (GINI), las cuales se miden como sigue:

La variable ILE, cuya fórmula de cálculo es:

$$
I L E_{t}=\left[\frac{2}{3}\left(\frac{T A_{t}}{100}\right)+\frac{1}{3}\left(\frac{\text { Mediana }_{t}}{15}\right)\right]
$$

donde:

$\mathrm{TA}=$ Tasa de alfabetismo en Colombia.

Mediana $=$ Nivel de educación alcanzado por el 50\% de la población (mediana de la educación).

Los valores de la variable ILE se obtienen del Ministerio de Educación Nacional (2008). Si el coeficiente de ILE es positivo, entonces se evidencia el impacto favorable que ejerce la educación en el crecimiento de las empresas por tamaño. De manera similar, los datos de la variable GINI referentes al período de estudio se adquieren de Informes de Desarrollo Humano 1991-2009 del Plan de las Naciones Unidas para el Desarrollo (PNUD). Un coeficiente de GINI negativo implica que mientras más inequitativa sea la sociedad menores tasas de crecimiento industrial registrarán las Mipymes del sector metalmecánico. 


\subsection{Variables externas}

La variable tasa de apertura a las exportaciones (TAE) indica el porcentaje de la producción que se exporta y se calcula como:

$$
T A E_{i j t}=\left[\frac{X_{i j t} / I P C}{Y_{i j t} / I P P}\right]
$$

donde:

$\mathrm{X}_{i j t}=$ Exportaciones del grupo industrial por tamaño de empresa.

La TAE se expresa como porcentaje. Mientras más alto sea su valor, mayor parte de la producción bruta real se destina al mercado externo. Dicho de otro modo, mientras más alta sea la TAE, la vocación exportadora del grupo industrial por tamaño de empresa es mayor y viceversa. Si el coeficiente de TAE es positivo un mayor volumen de exportaciones contribuirá con mayores tasas de CI.

Por otra parte, la variable tasa de penetración de las importaciones (TPI) mide la competitividad interna de un determinado grupo industrial del sector metalmecánico. Se expresa como el cociente entre las importaciones de materias primas, materiales y empaques sobre el consumo aparente (CA).

$$
T P I_{i j t}=\left[\frac{I_{i j t} / I P C}{C A_{i j t}}\right]
$$

donde:

$\mathrm{I}_{i j t}=$ Importaciones de materias primas, materiales y empaques del grupo industrial por tamaño de empresa.

$$
C A_{i j t}=\left[\frac{Y_{i j t} / I P P}{I_{i j t} / I P C}\right]-\left(X_{i j t} / I P C\right)
$$

La TPI varía entre 0 y 100\%. Cuanto mayor sea el porcentaje, menor será la capacidad por tamaño de empresa de abastecer su demanda interna con la producción nacional. Un coeficiente de TPI $<0$ implica que cuanto más dependan de insumos foráneos las Mipymes, menor será su crecimiento. 
Una vez obtenida la TPI, es posible calcular la variable tasa de exposición a la competencia internacional (TE):

$$
T E_{i j t}=\left[\frac{X_{i j t} / I P C}{Y_{i j t} / I P P}\right]+\left[1-\frac{X_{i j t} / I P C}{Y_{i j t} / I P P}\right] * T P I_{i j t}
$$

Esta variable representa el porcentaje en que la producción bruta real por tamaño de empresa está expuesta a la competencia internacional. Es decir, las ventas netas de productos fabricados por tamaño de empresa en otros países están sujetas a la competencia externa y la producción bruta real destinada al consumo interno está sujeta a la TPI. Mientras más cercana a uno sea la variable, se interpretará que las empresas por tamaño se encuentran cada vez más expuestas a la competencia externa. A su vez, si la TE se aproxima a cero, se entenderá que la producción interna tiene una baja exposición a la competencia internacional, bien por el lado de las exportaciones o por el de las importaciones. Un coeficiente negativo de TE significa que una mayor exposición de las MiPyME a la competencia externa afectará en forma desfavorable el crecimiento sectorial.

\subsection{Variable institucional}

Como variable institucional se toma el índice de percepción de la corrupción, que sería una variable proxy (Corrupción) del desempeño institucional del gobierno colombiano entre 2002 y 2006, correspondiente a la primera administración del presidente Álvaro Uribe Vélez. Este índice mide, en una escala de cero (percepción de muy corrupto) a diez (percepción de ausencia de corrupción) los niveles de percepción de la corrupción en el sector público de un país. La fuente de esta variable es la organización Transparencia Internacional. Si el coeficiente de Corrupción es negativo, indica que mayores niveles de corrupción estatal provocarán un descenso en la tasa de crecimiento de las Mipymes del sector metalmecánico.

\subsection{El modelo}

A continuación se presenta una descripción del modelo econométrico utilizado y la metodología asociada a la estimación. El modelo a estimar es el siguiente:

$$
\begin{aligned}
& y_{i t}=y_{i t-1} \gamma+x_{i t} \beta+u_{i}+\varepsilon_{i t} \\
& \text { donde } \\
& i \quad=1,2 \ldots \mathrm{N} ; t=1,2, \ldots \mathrm{T}
\end{aligned}
$$


$y_{i t}=$ variable endógena.

$y_{i t-1}=$ variable endógena rezagada 1 período.

$\chi_{i t}=$ vector de covariables exógenas.

$\beta=$ vector de parámetros a estimar.

$u_{i}=$ efectos individuales que capturan la heterogeneidad inobservada.

$\varepsilon_{\text {it }} \quad=$ error aleatorio.

Se trata de un modelo de datos de panel dinámico. Por construcción, $y_{i t-1}$ está correlacionado con el efecto individual no observado $u_{i}$. En consecuencia, se toman las primeras diferencias en ambos lados y se buscan las variables instrumentales para obtener los estimadores por el Método Generalizado de Momentos (MGM) propuesto por Arellano y Bond (1991).

La primera diferenciación de la ecuación [1] produce:

$$
\Delta y_{i t}=\Delta y_{i t-1} \gamma+\Delta x_{i t} \beta+\Delta \varepsilon_{i t}
$$

En la ecuación [2], los $u_{i t}$ desaparecen, pero el $y_{i t-1}$ en $\Delta y_{i t-1}$ es una función del $\varepsilon_{i t-1}$, el cual está también incorporado en $\Delta \varepsilon_{i t}$. De este modo, $\Delta y_{i t-1}$ está correlacionada con $\Delta \varepsilon_{i t}$ por construcción.

Arellano y Bond (1991) muestran cómo construir estimadores basados en ecuaciones de momentos construidas con niveles rezagados de la variable $y_{i t}$ y los errores en primeras diferencias. Si $\varepsilon_{i t}$ es idéntico e independientemente distribuido en $i$ y $t$, es decir, no hay correlación serial en los errores, un instrumento válido para $\Delta y_{i t-1}$ será $\Delta y_{i t-2}$. En tal caso, $\Delta y_{i t-2}$ está correlacionado con $\Delta y_{i t-1}=\Delta y_{i t-1-} \Delta y_{i t-2,}$ pero no está correlacionado con $\Delta \varepsilon_{i t}=\varepsilon_{i t}-\varepsilon_{i t-1}$. De hecho, se consideran instrumentos válidos cualesquiera valores de $y_{i t}$ rezagados (s) dos períodos o más.

$$
E\left[\left(\begin{array}{c}
y_{i t-2} \\
y_{i t-3} \\
\vdots \\
y_{i t}
\end{array}\right) \Delta \varepsilon_{i t}\right]=0 \Leftrightarrow E\left[y_{i s} \Delta \varepsilon_{i t}\right]=0, \quad s \leq t-2
$$

De esta forma, el MGM permite obtener el estimador asintóticamente más eficiente al usar todos los rezagos de $y_{i t}\langle\langle$ v15n32a06el3.wmf $\rangle\rangle$ posibles. La estimación del modelo dinámico se lleva a cabo sin recurrir a instrumentos externos. El modelo puede incluir regresores (estrictamente) exógenos $\left.\mathrm{El}_{i t} \mid u_{i}=x_{j, i 1}, \ldots, x_{j, i \mathrm{~T}}\right\rfloor=$ 
0 , al utilizar sus propios instrumentos $\mathrm{E}\left[\chi_{i, i t} \varepsilon_{i t}\right]=0$, o también todos sus rezagos y adelantos $E\left[x_{j, i} \varepsilon_{i t}\right]=0, s \neq t$.

Asimismo, es posible generalizar el argumento de rezagos de $y_{i t-1}$ como instrumentos a otros regresores que no sean estrictamente exógenos sin necesidad de buscar nuevos instrumentos. Por ejemplo, si se tienen regresores que, como $y_{i t-1}$, son predeterminados (débilmente exógenos) $\mathrm{E}\left[\varepsilon_{i t} \mid u_{i}=x_{j, i 1}, \ldots, x_{j, i t}\right]$ $=0$, entonces deben estar correlacionados con los errores pasados $E\left[x_{j, i t i s}\right] \neq 0$, $s<t\langle\langle v 15 n 32 a 06 e 14$.wmf $\rangle\rangle$, pero no con errores futuros $E\left[x_{j, i t} \varepsilon_{i s}\right] \neq 0, s \geq t$. Algunos regresores pueden ser contemporáneamente endógenos $E\left[x_{i, i t i s}\right] \neq 0, s \leq t$ pero no correlacionados con los errores futuros $\left.\mathrm{E}\left[\chi_{j, i t} \varepsilon_{i s}\right] \neq 0, s\right\rangle t$.

Con los supuestos anteriores se garantiza que el estimador MGM es consistente y asintóticamente normal y eficiente. El modelo a estimar es el siguiente:

$$
\Delta C I_{i t}=C I_{i t-1} \gamma+x_{i t}^{\prime} \beta+\delta_{i}+u_{i}+\varepsilon_{i t}
$$

donde

$$
\begin{aligned}
i= & 1, \ldots, \mathrm{N} ; t=2, \ldots, \mathrm{T} \\
\Delta \mathrm{Cl}_{i t}= & \text { tasa de crecimiento de la producción bruta del tamaño de empresa } i \text { en } \\
& \text { el período } t . \\
\mathrm{Cl}_{i t-1}= & \text { tasa de crecimiento de la producción bruta del tamaño de empresa } i \text { en } \\
& \text { el período } t-1 . \\
x_{i t}= & \text { vector de variables explicativas relacionadas con el tamaño de empresa } \\
& i \text { en el período } t . \\
\delta_{i=}= & \text { efecto temporal que captura efectos inobservables comunes a todos } \\
& \text { los tamaños de empresa. }
\end{aligned}
$$

Al estimar los parámetros de la ecuación [3] con MGM en primeras diferencias, esta se transforma así:

$$
\Delta C I_{i t}=\Delta C I_{i t-1} \gamma+\Delta x_{i t}^{\prime} \beta+\Delta \delta_{i}+\varepsilon_{i t}
$$

donde $i=1, \ldots, \mathrm{N} ; t=2, \ldots, \mathrm{T}$

Luego de la estimación se realiza la prueba de Sargan para contrastar la validez de los instrumentos. La estimación de los modelos se llevó a cabo en el programa STATA 12. 
Determinantes del crecimiento de las micro, pequeñas y medianas empresas colombianas: el caso del sector metalmecánico

\section{RESULTADOS ECONOMÉTRICOS}

Siguiendo la metodología explicada en la sección precedente, se estimaron los modelos para cada tamaño de empresa. Las versiones finales de los modelos solo incluyen las variables que resultaron ser estadísticamente significativas. En particular, las variables ILE y GINI reportaron una alta colinealidad entre sí. Por tal razón, se optó por incluir aquella que presentó mayor significancia estadística. Vale la pena señalar, además, que la inclusión de la variable $\mathrm{CI}_{t-1}$ proporcionó un mejor ajuste al resto de variables explicativas en cada una de las estimaciones realizadas. Las variables RKP, TAE y TPM no resultaron ser estadísticamente significativas en ninguno de los tamaños de empresa.

\subsection{Microempresas}

En la tabla 5, se observa convergencia en el crecimiento de las microempresas, a juzgar por el signo del coeficiente estimado de la variable $\mathrm{Cl}_{t-1}$, aunque esta no resultó ser estadísticamente significativa.

Tabla 5. Modelo estimado para las microempresas

\begin{tabular}{|l|c|c|}
\hline \multicolumn{1}{|c|}{ Variable } & Coeficiente & Error estándar robusto \\
\hline $\mathrm{CI}_{t-1}$ & -0.06 & 0.11 \\
\hline SPI & $3.25 \mathrm{e}-06^{*}$ & $5.56 \mathrm{e}-07$ \\
\hline CLU & $-98.21^{*}$ & 22.67 \\
\hline ICL & $-10.57^{*}$ & 3.25 \\
\hline ILE & $-520.30^{* *}$ & 246.30 \\
\hline Corrupción & $-72.20^{*}$ & 13.81 \\
\hline Constante & $698.09^{*}$ & 242.47 \\
\hline
\end{tabular}

* Estadísticamente significativo al $1 \%$

** Estadísticamente significativo al 5\%

Estadístico de Wald $(\chi 2)=136.68$

Probabilidad $(\chi 2)=0.00$

Observaciones $=20$

Grupos $=10$

Fuente: elaboración propia.

El coeficiente positivo de la variable SPI establece que, al mantener los demás factores constantes, un incremento del promedio de los salarios generó crecimiento industrial en las microempresas durante el período de estudio. Por su parte, el coeficiente estimado correspondiente a la variable CLU evidencia el hecho de que, 
desde la perspectiva del microempresario, un aumento del costo de la mano de obra requerido para producir una unidad de producto ocasionó una menor dinámica industrial. Además, el estimador de ICL señala que, ceteris paribus, la presencia de un mayor número de establecimientos redujo el $\mathrm{CI}$ de las microempresas.

Por otro lado, el coeficiente estimado de la variable ILE establece que en el caso de las microempresas, ceteris paribus, un mayor nivel de escolaridad impactó negativamente su CI. De forma similar, los mayores niveles de corrupción estatal infligieron un retroceso en el crecimiento de las microempresas durante el cuatrienio en estudio, dado el signo del coeficiente estimado para la variable Corrupción.

\subsection{Pequeñas empresas}

Una vez seleccionado el modelo de regresión correspondiente a las pequeñas empresas se obtuvieron los resultados que proporciona la tabla 6. En primer lugar, se observa que el signo del estimador de la variable $\mathrm{Cl}_{t-1}$ revela un proceso de convergencia entre las pequeñas empresas durante el periodo observado.

Tabla 6. Modelo estimado para las pequeñas empresas

\begin{tabular}{|l|c|c|}
\hline \multicolumn{1}{|c|}{ Variable } & Coeficiente & Error estándar robusto \\
\hline CIt-1 & $-0.67^{*}$ & 0.18 \\
\hline SPI & $1.83 \mathrm{e}-06^{* * *}$ & $1.08 \mathrm{e}-06$ \\
\hline CLU & $-208.86^{*}$ & 68.04 \\
\hline ICL & $-14.61^{* *}$ & 7.20 \\
\hline TE & $-1.14^{* *}$ & 0.53 \\
\hline Constante & $34.19^{*}$ & 9.99 \\
\hline
\end{tabular}

* Estadísticamente significativo al $1 \%$

* Estadísticamente significativo al 5\%

$*$ Estadísticamente significativo al 10\%

Estadístico de Wald $(\chi 2)=31.90$

Probabilidad $(\chi 2)=0.00$

Observaciones $=24$

Grupos $=12$

Fuente: elaboración propia.

El coeficiente estimado de la variable SPI muestra la respuesta positiva que tuvo el CI ante mayores niveles salariales. Mientras que el estimador de la variable CLU sugiere que un costo más alto del trabajo requerido para producir una unidad de producto implicó un CI menor. Estos dos últimos resultados reflejan el hecho de 
Determinantes del crecimiento de las micro, pequeñas y medianas empresas colombianas: el caso del sector metalmecánico

que si bien un salario mayor incentiva a los trabajadores a ser más productivos, el encarecimiento del factor trabajo podría hacer que los empresarios estén menos dispuestos a contratarlos. El signo negativo del estimador de ICL implica que frente a una mayor competencia local, las pequeñas empresas registraron un menor crecimiento. El signo negativo del estimador de TE, a su turno, implica que cuanto más se expuso la producción bruta real de las pequeñas empresas a la competencia externa, menor fue el crecimiento que registraron en el período analizado.

\subsection{Medianas empresas}

Con base en la tabla 7 se observa que las medianas empresas convergen hacia el estado estacionario, dado el coeficiente de $\mathrm{CI}_{t-1}$.

Tabla 7. Modelo estimado para las medianas empresas

\begin{tabular}{|l|c|c|}
\hline \multicolumn{1}{|c|}{ Variable } & Coeficiente & Error estándar robusto \\
\hline CIt-1 & $-0.52^{*}$ & 0.20 \\
\hline SPI & $5.92 \mathrm{e}-07^{*}$ & $2.04 \mathrm{e}-07$ \\
\hline CLU & $-55.62^{*}$ & 19.22 \\
\hline ICL & $-2.02^{* *}$ & 1.05 \\
\hline Constante & $3.47^{* *}$ & 1.82 \\
\hline
\end{tabular}

* Estadísticamente significativo al $1 \%$

* Estadísticamente significativo al 10\%

Estadístico de Wald $(\chi 2)=34.42$

Probabilidad $(\chi 2)=0.00$

Observaciones $=20$

Grupos $=10$

Fuente: elaboración propia.

En ese orden, el estimador de SPI denota la relación positiva que mantuvo el promedio salarial con el $\mathrm{Cl}$ entre las medianas empresas. Por el contrario, el coeficiente estimado de la variable CLU señala que los altos costos laborales condujeron a un menor crecimiento de estas empresas. Finalmente, el signo del estimador de ICL advierte que la competencia local originó efectos negativos en el CI de las medianas empresas.

\section{DISCUSIÓN}

Las micro, pequeñas y medianas empresas juegan un rol importante en el crecimiento del sector metalmecánico colombiano. En este artículo se han querido mostrar los 
determinantes del crecimiento sectorial conforme a esta tipología de empresas. Los resultados evidencian a grandes rasgos que el crecimiento de las micro, pequeñas y medianas empresas del sector está fuertemente influenciado por la competencia local. En tal sentido, los resultados encontrados para la concentración industrial confirman la tesis de Acs y Audretsch (1994): una mayor competencial local obstaculiza los procesos de innovación tecnológica que generan crecimiento industrial.

Otro de los hechos sobresalientes en relación con las variables salario promedio industrial y costo laboral unitario es que en todos los tamaños de empresa los resultados respaldan la teoría hicksiana de los salarios. En tal circunstancia, el sector mejoraría su competitividad ya sea con la disminución de los costos laborales por persona empleada o con el incremento de la productividad laboral.

Los resultados de la incidencia del logro educativo en el crecimiento de las micro, pequeñas y medianas empresas sectoriales es un asunto de debate. Así como se pone de manifiesto en los estudios de Benhabib y Spiegel (1994), Barro y Sala-i-Martin (1995), Bils y Klenow (1998) y Krueger y Lindahl (2001), el cambio en la educación no necesariamente es un determinante del crecimiento económico. Esto permite explicar el impacto negativo que puede ejercer la escolaridad sobre el crecimiento de la producción en las microempresas. Acemoglu y Zilibotti (2001) postulan que los avances tecnológicos que se generan en los países desarrollados se complementan con mano de obra calificada con el fin de aumentar su productividad. Para el caso del Reino Unido, por ejemplo, Sena y Higon (2012) muestran que las pequeñas y medianas empresas se benefician más de los spillovers de la investigación y desarrollo si la fuerza de trabajo de la región donde se ubican posee un alto logro educativo. Sin embargo, Acemoglu y Zilibotti (2001) recalcan que los países en vía de desarrollo pueden no incrementar su productividad en razón a que la baja calidad de la capacitación laboral dificulta la adopción de nuevas tecnologías.

Cobra importancia la complementariedad entre los resultados obtenidos en términos de la competitividad interna, el logro educativo y el cambio tecnológico. En los casos de las micro, pequeñas y medianas empresas del sector metalmecánico, se encuentra que la competencia local inhibe su crecimiento. Esto indica que no hay evidencia de un proceso de aglomeración industrial que contribuya a generar las condiciones propicias para el desarrollo tecnológico de las micro, pequeñas y medianas empresas del sector. Cabe resaltar, la precaria actuación de las universidades y los centros de investigación y desarrollo como fuentes de información para innovar. Este argumento se asocia al frágil nexo instituciones-sector productivo común en la mayoría de los sistemas de innovación de América Latina (Arocena y Sutz, 2002). Al respecto, ya algunos países en desarrollo son conscientes de esta problemática y están comenzando a aplicar paliativos. En Nigeria, por ejemplo, ya 
Determinantes del crecimiento de las micro, pequeñas y medianas empresas colombianas: el caso del sector metalmecánico

existe preocupación por la medición de la influencia de las capacidades tecnológicas de las pequeñas y medianas empresas metalmecánicas aglomeradas en los procesos de innovación e imitación de productos (Sobank, Ilori y Adegbite, 2012).

En relación con las variables de competitividad externa, los resultados para el caso de las pequeñas empresas confirman que los productos del sector metalmecánico, considerados bienes transables, son altamente vulnerables al ingreso de este tipo de mercancías foráneas al mercado doméstico. Este hecho no pasa desapercibido por el gremio del sector. Las micro, pequeñas y medianas empresas no cuentan con proyectos de investigación, ignoran los procedimientos para lograr el desarrollo tecnológico y tampoco se desenvuelven en espacios que les permitan planificar proyectos de índole tecnológica (Metal Actual, 2011). Quizá esto explique por qué la relación capital-producto, proxy del cambio tecnológico, no resulta ser estadísticamente significativa para ningún tamaño de empresa.

Cabe anotar que los resultados aquí encontrados no son definitivos. Al momento de realizar este estudio solo estaban disponibles los datos desagregados para las micro, pequeñas y medianas empresas manufactureras entre los años 2002 y 2006. Esa es la razón que explica el bajo número de observaciones que alimentan los modelos estimados. En la medida en que las cifras para años posteriores estén disponibles, se exhorta a la comunidad académica a profundizar en la revisión de las conclusiones aquí arrojadas. Por último, se sugiere incluir otras variables explicativas como el acceso al crédito, la inseguridad ciudadana, así como el aporte del empresariado local en el desarrollo de estas empresas.

\section{BIBLIOGRAFÍA}

Acemoglu, Daron y Zilibotti, Fabrizio (2001). Productivity Differences. En: The Quarterly Journal of Economics, Vol. 116, N. . 2, pp. 563-606.

Acs, Zoltan y Audretsch, David B (1994). Innovación, estructura del mercado y tamaño de la empresa. En: Desarrollo y gestión de PyMEs: aportes para un debate necesario. Universidad Nacional de General Sarmiento. Cap. 4.

Acs, Zoltan y Kallas, K. (2007). State of Literature on Small and Medium-Size Enterprises and Entrepreneurship in Low-Income Communities. Max Planck Institute of Economics, Discussion Papers on Entrepreneurship, Growth and Public Policy, N. ${ }^{\circ}$ 307, pp. 1-45.

Aghion, Philippe y Howitt, Peter (1997). Endogenous Growth Theory. Cambridge, MA: The MIT Press, 708p.

Aghion, Philippe (2002). Schumpeterian Growth Theory and the Dynamics of Income Inequality. En: Econometrica, Vol. 70, N. ${ }^{\circ}$ 3, pp. 855-882. 
Juan Carlos Trujillo Lora • Wilman Iglesias Pinedo

Amal, Mohamed y Rocha, Alexandre (2010). Internationalization of small -and mediumsized enterprises: a multi case study. En: European Business Review, Vol. 22, N. ${ }^{\circ}$ 6, pp. 608-623.

Archibugil, Daniele y Michie, Jonathan (1998). Technical change, Growth and Trade: New Departures in Institutional Economics. En: Journal of Economic Surveys, Vol. 12 N. 3 , pp. 1-20.

Arellano, Manuel y Bond, Stephen (1991). Some Tests of Specification for Panel Data: Monte Carlo Evidence and Application to Employment Equations. En: The Review of Economic Studies, Vol. 58, N. 2, pp. 277-297.

Arocena, R. y Sutz, J. (2002). Innovation Systems and Developing Countries. [En línea] DRUID Working Paper, 02 (05), University of Aalborg, Denmark. Disponible en: http://www.druid.dk/wp/pdf_files/02-05.pdf.

Arrow, Kenneth (1962). The Economic Implications of Learning by Doing. En: The Review of Economic Studies, Vol. 29, N. 3, pp. 155-173.

Arrow, Kenneth (1994). The production and distribution of knowledge. En: Silverberg G. y Soete, L. (eds.), The Economic of Growth and Technical Change. Technologies, Nations, Agents. Aldershot: Edward Elgar, pp. 9-19.

Barro, Robert (1991). Economic Growth in a Cross Section of Countries. En: The Quarterly Journal of Economics, Vol. 106, N. 2 2, pp. 407-443.

Barro, Robert y Sala-i-Martin, Xavier (1992). Convergence. En: Journal of Political Economy, Vol. 100, N. ${ }^{\circ}$, pp. 223-251.

Barro, Robert y Sala-i-Martin, Xavier (1995). Economic Growth. New York: McGraw-Hill, 539p.

Bell, Martin y Pavitt, Keith (1993). Technological accumulation and industrial growth: contrasts between developed and developing countries. En: Industrial and Corporate Change, Vol. 2, N. ${ }^{\circ}$, pp. 157-209.

Benhabib, Jess y Spiegel, Mark (1994). The Role of Human Capital in Economic Development: Evidence from Aggregate Cross-Country Data. En: Journal of Monetary Economics, Vol. 34, N. $^{\circ}$, pp.143-174.

Bils, Mark y Klenow, Peter (1998). Does Schooling Cause Growth?. En: The American Economic Review, Vol. 90, N. 5, pp. 1169-1183.

Cardona, Marlenny y Cano, Carlos (2005). La dinámica industrial, crecimiento económico y PyMEs: Un análisis de Datos de Panel para el caso colombiano 1980 -2000. Archivos de Economía, Documento 292. Bogotá: Departamento Nacional de Planeación, Septiembre. 50p. 
Determinantes del crecimiento de las micro, pequeñas y medianas empresas colombianas: el caso del sector metalmecánico

Cheng, H. y Yu, C. (2008). Institutional pressures and initiation of internationalization: evidence from Taiwanese small -and medium-sized enterprises. En: International Business Review, Vol. 17, pp. $331-48$.

CRC -Congreso de la República de Colombia- (1960). Ley 81. Ley orgánica del impuesto sobre la renta. Bogotá, Diario Oficial, año XCVII, No 30.412, 24 de diciembre de 1960, p. 539.

CRC -Congreso de la República de Colombia - (2000). Ley 590. Por la cual se dictan disposiciones para promover el desarrollo de las micro, pequeñas y medianas empresa. Bogotá, Diario Oficial 44.078, 10 de julio de 2000, 39p.

CRC -Congreso de la República de Colombia- (2004). Ley 950. Por medio de la cual se modifica la Ley 590 de 2000 sobre promoción del desarrollo de la micro, pequeña y mediana empresa colombiana y se dictan otras disposiciones. Bogotá, Diario Oficial 45.628, 02 de agosto de 2004, 21p.

Deininger, Klaus y Squire, Lyn (1998). New ways of looking at old issues: inequality and growth. En: Journal of Development Economics, Vol. 57, pp. 259-287.

Easterly, William (2002). The Elusive Quest for Growth. Cambridge, MA: The MIT Press. 356p.

Freeman, C. (1994). The economics of technical change. En: Cambridge Journal of Economics, Vol. 18, N. ${ }^{\circ}$, pp. 463-514.

Garay, Luis (2004). Colombia estructura industrial e internacionalización 1967-1996. [En línea] Publicación digital en la página web de la Biblioteca Luis Ángel Arango del Banco de la República. Disponible en: http://www.banrepcultural.org/blaavirtual/economia/ industrilatina/indice.htm. Búsqueda realizada en septiembre 5 de 2005.

González, Francisco; Guzmán, Carolina y Pachón, Ángela (1998). Productividad y retornos sociales del capital humano: Microfundamentos y evidencia para Colombia. Archivos de Macroeconomía, Documento 98. Bogotá: Departamento Nacional de Planeación, Noviembre. 37p.

Gould, D. M., y Ruffin, R. J. (1995). Human Capital, Trade, and Economic Growth. En: Review of World Economics, Vol. 131, N. ${ }^{\circ}$ 3, pp. 425-445.

Grossman, Gene y Helpman, Elhanan (1994). Endogenous Innovation in the Theory of Growth. En: The Journal of Economic Perspectives, Vol. 8, N. ${ }^{\circ}$ 1, pp. 23-44.

Guterman, Lia (1996). Colombia: Estrategias de Competitividad, Productividad, Recursos Humanos y Empleo en la Industria Metalmecánica. [En línea] Organización Internacional del Trabajo (OIT) y Agencia Canadiense para el Desarrollo Internacional (ACDI). Disponible en: http://www.oit.org.pe/WDMS/bib/publ/doctrab/dt_049.pdf. 45p.

Hicks, J. R. (1977). Valor y Capital. México: Fondo de Cultura Económica. 422p. 
Juan Carlos Trüillo Lora • Wilman Iglesias Pinedo

Informes de Desarrollo Humano 1991-2009. [En líneal PNUD. Disponible en: http://www. saludcolombia.com/actual/Salud\%2084/Colombia\%20IDH.pdf.

Katz, Jorge (1983). Cambio tecnológico en la industria metalmecánica latinoamericana. Resultados de un programa de estudios de casos. En: Revista de la CEPAL, N. ․ 19, pp. 87- 146.

Krueger, Alan y Lindahl, Mikael (2001). Education for Growth: Why and For Whom?. En: Journal of Economic Literature, Vol. XXXIX, pp. 1101-1136.

Kuznets, Simon (1955). Economic Growth and Income Inequality. En: The American Economic Review, Vol. 45, N.1, pp. 1-28.

Lall, Sanjaya (1992). Technological Capabilities and Industrialization. En: World Development, Vol. 20, ‥ 2, pp. 165-186.

Lucas, Robert (1988). On the mechanics of economic development. En: Journal of Monetary Economics, Vol. 22, pp. 3-42.

Lundvall, Bengt-Ake. (1992). National systems of innovation. Towards a theory of innovations and interactive learning. London: Pinter Publishers, 342p.

Metal Actual (2011). [En línea] Consulta sectorial. Industria Metalmecánica Frente a la Investigación y el Desarrollo. Disponible en: http://www.metalactual.com/revista/19/ iniciales_19.pdf.

Ministerio de Educación Nacional (2008). [En línea] Revolución Educativa 2002-2005. Disponible en: http://www.colombiaaprende.edu.co/html/home/1592/article-91697.html.

Nelson, Richard y Phelps, Edmund (1966). Investment in Humans, Technological Diffusion, and Economic Growth. En: The American Economic Review, Vol. 56, N.1/2, pp. 69-75.

North, Douglass (1994). Economic Performance Through Time. En: The American Economic Review, Vol. 84, N. ${ }^{\circ}$ 3, pp. 359-368.

Porter, Michael (1991). La ventaja competitiva de las naciones. Buenos Aires: Vergara, 1025p.

Poveda, Gabriel (1970). Historia de la industria en Colombia. En: Revista Trimestral de la Asociación Nacional de Industriales (ANDI), No. 11. Medellín: Editorial Bedout S. A., 97p.

Poveda, Gabriel (1990). Comentario al documento "Experiencias de Reestructuración Industrial" de Juan José Echavarría. [En línea] Publicación digital en la página web de la Biblioteca Luis Ángel Arango del Banco de la República. Disponible en: http:// banrepcultural.org/blaavirtual/ciencias/sena/metalurgia/fedemetal/fedeme5b.htm. Búsqueda realizada en octubre 12 de 2011.

Rebelo, Sergio (1991). Long-Run Policy Analysis and Long-Run Growth. En: Journal of Political Economy, Vol. 99, N. ${ }^{\circ}$, pp. 500-521. 
Determinantes del crecimiento de las micro, pequeñas y medianas empresas colombianas: el caso del sector metalmecánico

Romer, Paul (1986). Increasing Returns and Long-Run Growth. En: The Journal of Political Economy, Vol. 94, N. ${ }^{\circ}$ 5, pp. 1002-1037.

Romer, Paul (1990). Endogenous Technological Change. En: The Journal of Political Economy, Vol. 98, No. 5, Part 2: The Problem of Development: A Conference of the Institute for the Study of Free Enterprise Systems, pp. S71-S102.

Sena, V. y Higon, D. A. (2012). Productivity, RED Spillovers and Educational Attainment. En: Oxford Bulletin of Economics and Statistics, 0305-9049. doi: 10.1111/j.1468-0084.2012.007 24.x. pp. 1-23.

Sobanke, Victor; Ilori, Matthew; y Adegbite, Stephen (2012). Technological Capability in Metal Fabricating Firms in Southwestern Nigeria. En: American Journal of Industrial and Business Management, 2, pp. 176-183.

Solow, Robert (1956). A Contribution to the Theory of Economic Growth. En: The Quarterly Journal of Economics, Vol. 70, N. 1 , pp. 65-94.

UNIDO y UNODC (2007). Fighting Corruption. Corruption Prevention to Foster SME Development. [En línea] United Nations Industrial Development Organization (UNIDO) y the United Nations Office on Drugs and Crime (UNODC). Disponible en: http://www.unido.org/ index.php?id=5651. $25 \mathrm{p}$.

Uzawa, Hirofumi (1965). Optimum Technical Change in an Aggregative Model of Economic Growth. En: International Economic Review, Vol. 6, N. ${ }^{\circ}$ 1, pp. 18-31.

Zevallos, Emilio (2003). Micro, pequeñas y medianas empresas en América Latina. En: Revista de la CEPAL, N. ${ }^{\circ}$ 79, pp. 53-70.

Zhu, H.; Hitt, M.; y Tihanyi, L. (2007). The internationalization of SMEs in emerging economies: institutional embeddedness and absorptive capacities. En: Journal of Small Business Strategy, Vol. 17, N. ${ }^{\circ} 2$, pp. 1-25. 
Juan Carlos Truïllo Lora • Wilman Iglesias Pinedo

ANEXOS

Anexo A.

Variables empleadas en la estimación de los modelos para las microempresas colombianas del sector metalmecánico, 2002-2006.

\begin{tabular}{|c|c|c|c|c|c|c|c|c|c|c|c|c|c|c|}
\hline Años & CIIU & $\mathrm{Cl}$ & SPI & RKP & koratio & CLU & ICL & ILE & $i d h$ & gini & TAE & TPI & TE & Corrup \\
\hline 2003 & 271 & 8,46 & 4.300 .000 & $34.091,4$ & 1,72 & 0,03 & 0,91 & 0,79 & 0,79 & 0,57 & 0,00 & 0,00 & 0,00 & 3,7 \\
\hline 2004 & 271 & 35,99 & 7.900 .000 & $1.630,1$ & 0,02 & 0,03 & 0,43 & 0,79 & 0,79 & 0,58 & 7,26 & 0,00 & 0,07 & 3,8 \\
\hline 2005 & 271 & $-4,45$ & 4.200 .000 & $28.144,7$ & 1,67 & 0,11 & 0,85 & 0,78 & 0,79 & 0,58 & 0,00 & 0,00 & 0,00 & 4,0 \\
\hline 2006 & 271 & 0,21 & 4.200 .000 & $27.869,3$ & 1,83 & 0,11 & 0,90 & 0,79 & 0,79 & 0,59 & 0,00 & 0,00 & 0,00 & 3,9 \\
\hline 2003 & 272 & 2,07 & 5.300 .000 & $21.029,2$ & 0,84 & 0,08 & 1,18 & 0,79 & 0,79 & 0,57 & 5,44 & 8,47 & 8,07 & 3,7 \\
\hline 2004 & 272 & 7,74 & 6.200 .000 & $3.240,5$ & 0,18 & 0,09 & 0,82 & 0,79 & 0,79 & 0,58 & 4,59 & 26,70 & 25,52 & 3,8 \\
\hline 2005 & 272 & $-3,88$ & 5.200 .000 & $11.599,1$ & 0,62 & 0,11 & 0,98 & 0,78 & 0,79 & 0,58 & 24,96 & 0,00 & 0,25 & 4,0 \\
\hline 2006 & 272 & $-0,28$ & 5.700 .000 & $15.079,3$ & 0,89 & 0,11 & 0,92 & 0,79 & 0,79 & 0,59 & 32,78 & 4,85 & 3,59 & 3,9 \\
\hline 2003 & 281 & 0,52 & 4.300 .000 & $34.032,0$ & 1,70 & 0,07 & 1,10 & 0,79 & 0,79 & 0,57 & 0,74 & 0,88 & 0,88 & 3,7 \\
\hline 2004 & 281 & 2,70 & 5.200 .000 & $5.747,4$ & 0,33 & 0,11 & 1,28 & 0,79 & 0,79 & 0,58 & 1,87 & 0,87 & 0,88 & 3,8 \\
\hline 2005 & 281 & $-2,13$ & 4.700 .000 & $35.483,4$ & 1,92 & 0,07 & 1,04 & 0,78 & 0,79 & 0,58 & 0,01 & 0,15 & 0,15 & 4,0 \\
\hline 2006 & 281 & $-0,20$ & 4.300 .000 & $30.860,5$ & 1,80 & 0,07 & 1,03 & 0,79 & 0,79 & 0,59 & 0,00 & 0,49 & 0,49 & 3,9 \\
\hline 2003 & 289 & 0,20 & 4.500 .000 & $24.256,2$ & 1,25 & 0,10 & 1,02 & 0,79 & 0,79 & 0,57 & 0,00 & 0,00 & 0,00 & 3,7 \\
\hline 2004 & 289 & 2,14 & 4.100 .000 & $3.580,8$ & 0,27 & 0,16 & 0,92 & 0,79 & 0,79 & 0,58 & 1,50 & 0,82 & 0,82 & 3,8 \\
\hline 2005 & 289 & $-2,27$ & 3.500 .000 & $22.168,1$ & 1,05 & 0,07 & 1,02 & 0,78 & 0,79 & 0,58 & 3,12 & 1,51 & 1,50 & 4,0 \\
\hline 2006 & 289 & $-0,11$ & 4.700 .000 & $39.099,6$ & 1,96 & 0,09 & 1,05 & 0,79 & 0,79 & 0,59 & 0,61 & 2,13 & 2,12 & 3,9 \\
\hline 2003 & 291 & 0,71 & 2.600 .000 & $29.699,3$ & 1,36 & 0,05 & 0,91 & 0,79 & 0,79 & 0,57 & 0,07 & 6,61 & 6,60 & 3,7 \\
\hline 2004 & 291 & 1,26 & 5.500 .000 & $4.352,3$ & 0,22 & 0,13 & 1,13 & 0,79 & 0,79 & 0,58 & 2,55 & 3,71 & 3,64 & 3,8 \\
\hline 2005 & 291 & $-1,43$ & 5.100 .000 & $28.785,0$ & 1,20 & 0,07 & 0,99 & 0,78 & 0,79 & 0,58 & 0,27 & 0,04 & 0,04 & 4,0 \\
\hline 2006 & 291 & $-0,66$ & 4.700 .000 & $19.946,7$ & 1,19 & 0,11 & 0,95 & 0,79 & 0,79 & 0,59 & 17,49 & 0,00 & 0,17 & 3,9 \\
\hline 2003 & 292 & $-0,11$ & 4.900 .000 & $20.739,6$ & 1,07 & 0,10 & 1,03 & 0,79 & 0,79 & 0,57 & 0,11 & 9,79 & 9,78 & 3,7 \\
\hline 2004 & 292 & 2,52 & 5.000 .000 & $6.420,9$ & 0,36 & 0,13 & 1,28 & 0,79 & 0,79 & 0,58 & 1,96 & 0,40 & 0,41 & 3,8 \\
\hline 2005 & 292 & $-1,11$ & 5.000 .000 & $25.661,3$ & 1,14 & 0,09 & 1,02 & 0,78 & 0,79 & 0,58 & 5,92 & 0,46 & 0,50 & 4,0 \\
\hline 2006 & 292 & 0,33 & 4.800 .000 & $24.352,2$ & 1,03 & 0,08 & 0,94 & 0,79 & 0,79 & 0,59 & 6,48 & 0,21 & 0,26 & 3,9 \\
\hline 2003 & 293 & $-0,76$ & 3.800 .000 & $20.739,6$ & 6,87 & 0,11 & 1,35 & 0,79 & 0,79 & 0,57 & 0,00 & 0,00 & 0,00 & 3,7 \\
\hline 2004 & 293 & $-0,03$ & 3.600 .000 & $6.420,9$ & 0,80 & 0,13 & 1,54 & 0,79 & 0,79 & 0,58 & 0,00 & 0,00 & 0,00 & 3,8 \\
\hline
\end{tabular}


Determinantes del crecimiento de las micro, pequeñas y medianas empresas colombianas: el caso del sector metalmecánico

\begin{tabular}{|c|c|c|c|c|c|c|c|c|c|c|c|c|c|c|}
\hline Años & CIIU & CI & SPI & RKP & koratio & CLU & ICL & ILE & idh & gini & TAE & TPI & TE & Corrup \\
\hline 2005 & 293 & $-0,76$ & 3.800 .000 & $25.661,3$ & 17,89 & 0,11 & 1,21 & 0,78 & 0,79 & 0,58 & 0,00 & 0,00 & 0,00 & 4,0 \\
\hline 2006 & 293 & $-0,03$ & 3.900 .000 & $24.352,2$ & 1,92 & 0,09 & 1,29 & 0,79 & 0,79 & 0,59 & 0,00 & 0,00 & 0,00 & 3,9 \\
\hline 2003 & 342 & 0,59 & 4.500 .000 & $10.958,1$ & 0,55 & 0,06 & 1,05 & 0,79 & 0,79 & 0,57 & 0,00 & 0,00 & 0,00 & 3,7 \\
\hline 2004 & 342 & 0,49 & 4.200 .000 & $5.386,2$ & 0,29 & 0,07 & 1,98 & 0,79 & 0,79 & 0,58 & 0,00 & 0,00 & 0,00 & 3,8 \\
\hline 2005 & 342 & 0,05 & 4.400 .000 & $8.334,8$ & 0,31 & 0,05 & 0,95 & 0,78 & 0,79 & 0,58 & 0,00 & 0,00 & 0,00 & 4,0 \\
\hline 2006 & 342 & $-0,12$ & 361.329 & $10.412,3$ & 0,42 & 0,00 & 1,01 & 0,79 & 0,79 & 0,59 & 0,00 & 0,33 & 0,33 & 3,9 \\
\hline 2003 & 343 & 0,59 & 4.400 .000 & $24.001,1$ & 1,57 & 0,15 & 0,86 & 0,79 & 0,79 & 0,57 & 2,95 & 0,40 & 0,42 & 3,7 \\
\hline 2004 & 343 & 2,34 & 4.500 .000 & $4.715,3$ & 0,32 & 0,13 & 1,32 & 0,79 & 0,79 & 0,58 & 2,18 & 2,30 & 2,27 & 3,8 \\
\hline 2005 & 343 & $-1,51$ & 5.000 .000 & $15.122,4$ & 0,90 & 0,13 & 0,94 & 0,78 & 0,79 & 0,58 & 1,47 & 3,17 & 3,14 & 4,0 \\
\hline 2006 & 343 & 0,91 & 5.000 .000 & $26.049,8$ & 0,86 & 0,08 & 1,08 & 0,79 & 0,79 & 0,59 & 0,83 & 1,90 & 1,89 & 3,9 \\
\hline 2003 & 359 & $-3,49$ & 1.600 .000 & $18.366,1$ & 1,24 & 0,03 & 1,05 & 0,79 & 0,79 & 0,57 & 0,00 & 0,00 & 0,00 & 3,7 \\
\hline 2004 & 359 & 2,08 & 642.438 & $7.502,2$ & 0,36 & 0,01 & 1,06 & 0,79 & 0,79 & 0,58 & 0,00 & 0,00 & 0,00 & 3,8 \\
\hline 2005 & 359 & $-3,49$ & 384.889 & $24.451,8$ & 1,74 & 0,01 & 1,06 & 0,78 & 0,79 & 0,58 & 0,00 & 0,00 & 0,00 & 4,0 \\
\hline 2006 & 359 & 2,08 & 3.700 .000 & $23.144,4$ & 1,62 & 0,08 & 1,04 & 0,79 & 0,79 & 0,59 & 0,00 & 0,00 & 0,00 & 3,9 \\
\hline
\end{tabular}

Fuente: elaboración propia

Anexo B.

Variables empleadas en la estimación de los modelos para las empresas pequeñas colombianas del sector metalmecánico, 2002-2006

\begin{tabular}{|c|c|c|c|c|c|c|c|c|c|c|c|c|c|c|}
\hline Años & CIIU & CI & SPI & RKP & koratio & CLU & ICL & ILE & idh & gini & TAE & TPI & TE & Corrup \\
\hline 2003 & 271 & 0,44 & 5.100 .000 & $29.320,7$ & 1,35 & 0,06 & 1,02 & 0,79 & 0,79 & 0,57 & 1,76 & 24,23 & 23,82 & 3,7 \\
\hline 2004 & 271 & $-0,95$ & 4.000 .000 & $17.562,9$ & 1,01 & 0,10 & 1,20 & 0,79 & 0,79 & 0,58 & 4,83 & 4,15 & 4,00 & 3,8 \\
\hline 2005 & 271 & 2,74 & 5.000 .000 & $32.779,9$ & 1,41 & 0,07 & 0,98 & 0,78 & 0,79 & 0,58 & 3,67 & 8,09 & 7,83 & 4,0 \\
\hline 2006 & 271 & $-0,08$ & 5.000 .000 & $24.839,2$ & 1,18 & 0,08 & 1,17 & 0,79 & 0,79 & 0,59 & 6,12 & 5,53 & 5,25 & 3,9 \\
\hline 2003 & 272 & 1,54 & 7.500 .000 & $35.508,2$ & 0,64 & 0,00 & 1,03 & 0,79 & 0,79 & 0,57 & 84,09 & 6,19 & 1,83 & 3,7 \\
\hline 2004 & 272 & $-1,90$ & 5.100 .000 & $22.070,2$ & 0,83 & 0,06 & 1,05 & 0,79 & 0,79 & 0,58 & 27,53 & 17,34 & 12,84 & 3,8 \\
\hline 2005 & 272 & 62,29 & 6.900 .000 & $25.641,9$ & 0,68 & 0,01 & 0,87 & 0,78 & 0,79 & 0,58 & 85,63 & 9,76 & 2,26 & 4,0 \\
\hline 2006 & 272 & 0,31 & 6.900 .000 & $28.048,8$ & 0,60 & 0,01 & 1,19 & 0,79 & 0,79 & 0,59 & 58,39 & 6,11 & 3,13 & 3,9 \\
\hline 2003 & 281 & 0,26 & 5.600 .000 & $37.583,6$ & 1,22 & 0,07 & 0,93 & 0,79 & 0,79 & 0,57 & 5,26 & 7,30 & 6,97 & 3,7 \\
\hline 2004 & 281 & 0,11 & 6.000 .000 & $19.046,2$ & 0,66 & 0,08 & 1,13 & 0,79 & 0,79 & 0,58 & 2,09 & 2,89 & 2,85 & 3,8 \\
\hline 2005 & 281 & 0,00 & 6.000 .000 & $23.598,8$ & 0,89 & 0,07 & 0,87 & 0,78 & 0,79 & 0,58 & 0,24 & 4,07 & 4,06 & 4,0 \\
\hline 2006 & 281 & 0,59 & 3.300 .000 & $15.929,8$ & 0,72 & 0,06 & 0,57 & 0,79 & 0,79 & 0,59 & 2,23 & 4,90 & 4,82 & 3,9 \\
\hline 2003 & 289 & 0,67 & 5.400 .000 & $27.467,2$ & 1,15 & 0,09 & 1,02 & 0,79 & 0,79 & 0,57 & 17,64 & 10,62 & 8,93 & 3,7 \\
\hline
\end{tabular}


Juan Carlos Trujillo Lora • Wilman Iglesias Pinedo

\begin{tabular}{|c|c|c|c|c|c|c|c|c|c|c|c|c|c|c|}
\hline Años & CIIU & $\mathrm{Cl}$ & SPI & RKP & koratio & CLU & ICL & ILE & $i d h$ & gini & TAE & TPI & TE & Corrup \\
\hline 2004 & 289 & 0,48 & 5.500 .000 & $17.828,8$ & 0,82 & 0,10 & 1,02 & 0,79 & 0,79 & 0,58 & 7,44 & 9,64 & 9,00 & 3,8 \\
\hline 2005 & 289 & 1,10 & 5.300 .000 & $27.282,0$ & 1,06 & 0,07 & 0,96 & 0,78 & 0,79 & 0,58 & 19,71 & 11,15 & 9,15 & 4,0 \\
\hline 2006 & 289 & 0,31 & 5.500 .000 & $25.346,3$ & 1,04 & 0,07 & 1,18 & 0,79 & 0,79 & 0,59 & 11,27 & 6,42 & 5,81 & 3,9 \\
\hline 2003 & 291 & 0,09 & 6.400 .000 & $34.977,0$ & 1,42 & 0,13 & 0,99 & 0,79 & 0,79 & 0,57 & 10,76 & 9,40 & 8,50 & 3,7 \\
\hline 2004 & 291 & 1,84 & 5.500 .000 & $18.340,9$ & 0,71 & 0,10 & 0,95 & 0,79 & 0,79 & 0,58 & 10,44 & 7,32 & 6,66 & 3,8 \\
\hline 2005 & 291 & $-1,22$ & 14.000 .000 & $60.382,9$ & 0,96 & 0,11 & 1,89 & 0,78 & 0,79 & 0,58 & 5,19 & 2,35 & 2,28 & 4,0 \\
\hline 2006 & 291 & 0,24 & 6.300 .000 & $24.927,3$ & 0,94 & 0,10 & 1,10 & 0,79 & 0,79 & 0,59 & 4,98 & 6,51 & 6,24 & 3,9 \\
\hline 2003 & 292 & 0,60 & 6.400 .000 & $30.205,9$ & 1,28 & 0,15 & 1,06 & 0,79 & 0,79 & 0,57 & 17,33 & 2,41 & 2,17 & 3,7 \\
\hline 2004 & 292 & 0,22 & 6.700 .000 & $21.560,2$ & 0,81 & 0,14 & 1,17 & 0,79 & 0,79 & 0,58 & 5,05 & 0,30 & 0,33 & 3,8 \\
\hline 2005 & 292 & $-0,04$ & 6.800 .000 & $31.034,0$ & 1,33 & 0,14 & 0,93 & 0,78 & 0,79 & 0,58 & 14,68 & 3,41 & 3,05 & 4,0 \\
\hline 2006 & 292 & 0,23 & 6.800 .000 & $30.799,2$ & 1,37 & 0,15 & 1,12 & 0,79 & 0,79 & 0,59 & 5,55 & 1,65 & 1,61 & 3,9 \\
\hline 2003 & 293 & 0,61 & 4.800 .000 & $19.230,6$ & 1,29 & 0,15 & 0,95 & 0,79 & 0,79 & 0,57 & 25,23 & 14,31 & 10,95 & 3,7 \\
\hline 2004 & 293 & 5,16 & 4.000 .000 & $11.152,4$ & 0,76 & 0,11 & 0,63 & 0,79 & 0,79 & 0,58 & 1,12 & 2,87 & 2,85 & 3,8 \\
\hline 2005 & 293 & $-2,84$ & 4.100 .000 & $13.030,7$ & 0,86 & 0,13 & 1,10 & 0,78 & 0,79 & 0,58 & 3,38 & 4,42 & 4,31 & 4,0 \\
\hline 2006 & 293 & $-0,49$ & 4.900 .000 & $10.206,0$ & 0,76 & 0,16 & 1,64 & 0,79 & 0,79 & 0,59 & 19,02 & 6,53 & 5,48 & 3,9 \\
\hline 2003 & 341 & 0,31 & 4.900 .000 & $38.739,3$ & 1,97 & 0,13 & 1,36 & 0,79 & 0,79 & 0,57 & 0,00 & 0,00 & 0,00 & 3,7 \\
\hline 2004 & 341 & 6,81 & 6.900 .000 & $21.783,2$ & 0,54 & 0,05 & 1,81 & 0,79 & 0,79 & 0,58 & 0,00 & 0,00 & 0,00 & 3,8 \\
\hline 2005 & 341 & $-3,24$ & 5.800 .000 & $47.076,5$ & 2,20 & 0,13 & 1,04 & 0,78 & 0,79 & 0,58 & 0,00 & 0,00 & 0,00 & 4,0 \\
\hline 2006 & 341 & 0,57 & 5.800 .000 & $38.422,4$ & 2,05 & 0,14 & 0,97 & 0,79 & 0,79 & 0,59 & 0,00 & 0,00 & 0,00 & 3,9 \\
\hline 2003 & 342 & 0,09 & 5.900 .000 & $44.112,2$ & 2,05 & 0,11 & 1,07 & 0,79 & 0,79 & 0,57 & 1,62 & 0,62 & 0,62 & 3,7 \\
\hline 2004 & 342 & 3,40 & 5.700 .000 & $13.634,9$ & 0,52 & 0,08 & 0,78 & 0,79 & 0,79 & 0,58 & 1,56 & 7,37 & 7,27 & 3,8 \\
\hline 2005 & 342 & $-2,47$ & 4.600 .000 & $12.822,0$ & 0,78 & 0,09 & 0,89 & 0,78 & 0,79 & 0,58 & 0,00 & 2,60 & 2,60 & 4,0 \\
\hline 2006 & 342 & 0,81 & 5.300 .000 & $11.372,6$ & 0,54 & 0,09 & 1,02 & 0,79 & 0,79 & 0,59 & 0,89 & 4,05 & 4,02 & 3,9 \\
\hline 2003 & 343 & $-0,05$ & 5.600 .000 & $23.322,6$ & 1,22 & 0,15 & 0,87 & 0,79 & 0,79 & 0,57 & 16,73 & 5,41 & 4,67 & 3,7 \\
\hline 2004 & 343 & 2,87 & 6.000 .000 & $14.964,5$ & 0,63 & 0,13 & 0,82 & 0,79 & 0,79 & 0,58 & 5,47 & 3,13 & 3,01 & 3,8 \\
\hline 2005 & 343 & $-1,67$ & 6.300 .000 & $25.286,0$ & 1,19 & 0,15 & 0,76 & 0,78 & 0,79 & 0,58 & 8,80 & 2,69 & 2,54 & 4,0 \\
\hline 2006 & 343 & $-0,31$ & 5.900 .000 & $25.964,5$ & 1,16 & 0,14 & 1,04 & 0,79 & 0,79 & 0,59 & 16,41 & 5,27 & 4,57 & 3,9 \\
\hline 2003 & 351 & $-0,22$ & 799.619 & $100.135,0$ & 3,74 & 0,01 & 1,07 & 0,79 & 0,79 & 0,57 & 16,93 & 0,00 & 0,17 & 3,7 \\
\hline 2004 & 351 & $-1,23$ & 3.900 .000 & $25.422,6$ & 1,87 & 0,12 & 1,03 & 0,79 & 0,79 & 0,58 & 0,00 & 0,00 & 0,00 & 3,8 \\
\hline 2005 & 351 & 19,44 & 7.300 .000 & $94.938,9$ & 3,03 & 0,07 & 0,75 & 0,78 & 0,79 & 0,58 & 1,59 & 0,00 & 0,02 & 4,0 \\
\hline 2006 & 351 & 0,10 & 807.330 & $186.000,0$ & 3,09 & 0,00 & 1,17 & 0,79 & 0,79 & 0,59 & 0,00 & 0,40 & 0,40 & 3,9 \\
\hline 2003 & 359 & $-0,15$ & 4.900 .000 & $12.105,3$ & 0,85 & 0,13 & 0,90 & 0,79 & 0,79 & 0,57 & 4,16 & 0,84 & 0,85 & 3,7 \\
\hline 2004 & 359 & 2,87 & 4.700 .000 & $9.885,1$ & 0,52 & 0,11 & 0,83 & 0,79 & 0,79 & 0,58 & 0,59 & 21,27 & 21,15 & 3,8 \\
\hline 2005 & 359 & $-1,50$ & 5.000 .000 & $10.708,5$ & 0,55 & 0,11 & 0,77 & 0,78 & 0,79 & 0,58 & 1,94 & 4,91 & 4,84 & 4,0 \\
\hline 2006 & 359 & 0,42 & 5.100 .000 & $13.849,3$ & 0,76 & 0,10 & 0,94 & 0,79 & 0,79 & 0,59 & 1,45 & 4,65 & 4,60 & 3,9 \\
\hline
\end{tabular}

Fuente: elaboración propia 
Determinantes del crecimiento de las micro, pequeñas y medianas empresas colombianas: el caso del sector metalmecánico

Anexo C.

Variables empleadas en la estimación de los modelos para las empresas medianas colombianas del sector metalmecánico, 2002-2006

\begin{tabular}{|c|c|c|c|c|c|c|c|c|c|c|c|c|c|c|}
\hline Años & CIIU & $\mathrm{Cl}$ & SPI & RKP & koratio & CLU & ICL & ILE & $i d h$ & gini & TAE & TPI & TE & Corrup \\
\hline 2003 & 271 & 0,36 & 8.700 .000 & $110.320,0$ & 1,81 & 0,05 & 0,88 & 0,79 & 0,79 & 0,57 & 6,39 & 25,00 & 23,46 & 3,7 \\
\hline 2004 & 271 & 0,34 & 6.200 .000 & $36.859,1$ & 0,63 & 0,03 & 0,92 & 0,79 & 0,79 & 0,58 & 3,66 & 5,88 & 5,70 & 3,8 \\
\hline 2005 & 271 & 0,30 & 7.000 .000 & $83.208,0$ & 2,10 & 0,04 & 0,90 & 0,78 & 0,79 & 0,58 & 15,60 & 38,03 & 32,25 & 4,0 \\
\hline 2006 & 271 & 0,26 & 6.300 .000 & $93.523,0$ & 2,25 & 0,03 & 0,95 & 0,79 & 0,79 & 0,59 & 14,03 & 10,22 & 8,93 & 3,9 \\
\hline 2003 & 272 & 0,26 & 4.600 .000 & $51.085,9$ & 2,17 & 0,06 & 1,14 & 0,79 & 0,79 & 0,57 & 30,34 & 9,60 & 6,99 & 3,7 \\
\hline 2004 & 272 & 0,48 & 4.600 .000 & $49.113,2$ & 1,34 & 0,05 & 1,33 & 0,79 & 0,79 & 0,58 & 14,67 & 4,04 & 3,59 & 3,8 \\
\hline 2005 & 272 & 2,67 & 4.900 .000 & $36.337,3$ & 1,03 & 0,05 & 0,98 & 0,78 & 0,79 & 0,58 & 17,32 & 2,25 & 2,03 & 4,0 \\
\hline 2006 & 272 & $-0,21$ & 6.000 .000 & $30.052,0$ & 1,03 & 0,06 & 1,09 & 0,79 & 0,79 & 0,59 & 12,68 & 9,46 & 8,39 & 3,9 \\
\hline 2003 & 281 & 1,09 & 6.000 .000 & $26.631,7$ & 0,76 & 0,07 & 1,01 & 0,79 & 0,79 & 0,57 & 16,94 & 9,82 & 8,33 & 3,7 \\
\hline 2004 & 281 & 0,10 & 5.400 .000 & $40.384,2$ & 1,04 & 0,05 & 1,56 & 0,79 & 0,79 & 0,58 & 7,14 & 13,63 & 12,73 & 3,8 \\
\hline 2005 & 281 & 2,98 & 5.400 .000 & $39.969,0$ & 1,08 & 0,05 & 0,97 & 0,78 & 0,79 & 0,58 & 5,65 & 16,76 & 15,87 & 4,0 \\
\hline 2006 & 281 & 0,13 & 5.000 .000 & $44.073,0$ & 1,11 & 0,04 & 1,04 & 0,79 & 0,79 & 0,59 & 7,36 & 9,48 & 8,86 & 3,9 \\
\hline 2003 & 289 & 0,55 & 7.500 .000 & $70.645,0$ & 1,71 & 0,08 & 1,01 & 0,79 & 0,79 & 0,57 & 14,28 & 22,11 & 19,10 & 3,7 \\
\hline 2004 & 289 & $-0,14$ & 6.600 .000 & $33.200,3$ & 0,96 & 0,09 & 0,86 & 0,79 & 0,79 & 0,58 & 16,42 & 9,93 & 8,46 & 3,8 \\
\hline 2005 & 289 & 1,48 & 7.100 .000 & $62.519,4$ & 1,50 & 0,07 & 0,88 & 0,78 & 0,79 & 0,58 & 9,67 & 15,59 & 14,18 & 4,0 \\
\hline 2006 & 289 & 0,14 & 7.200 .000 & $58.980,1$ & 1,50 & 0,08 & 0,96 & 0,79 & 0,79 & 0,59 & 11,86 & 11,12 & 9,92 & 3,9 \\
\hline 2003 & 291 & 0,11 & 7.100 .000 & $36.297,8$ & 1,04 & 0,10 & 0,98 & 0,79 & 0,79 & 0,57 & 21,65 & 16,20 & 12,91 & 3,7 \\
\hline 2004 & 291 & $-0,91$ & 6.700 .000 & $28.279,5$ & 1,04 & 0,12 & 0,92 & 0,79 & 0,79 & 0,58 & 19,05 & 16,00 & 13,14 & 3,8 \\
\hline 2005 & 291 & 5,90 & 17.000 .000 & $92.165,5$ & 0,99 & 0,08 & 2,37 & 0,78 & 0,79 & 0,58 & 12,45 & 10,83 & 9,60 & 4,0 \\
\hline 2006 & 291 & $-0,25$ & 6.000 .000 & $29.742,3$ & 0,89 & 0,08 & 1,02 & 0,79 & 0,79 & 0,59 & 14,50 & 17,99 & 15,53 & 3,9 \\
\hline 2003 & 292 & 0,69 & 9.100 .000 & $41.336,8$ & 1,05 & 0,14 & 1,14 & 0,79 & 0,79 & 0,57 & 23,46 & 12,41 & 9,73 & 3,7 \\
\hline 2004 & 292 & $-0,31$ & 8.300 .000 & $43.269,3$ & 1,33 & 0,13 & 1,80 & 0,79 & 0,79 & 0,58 & 10,37 & 6,94 & 6,33 & 3,8 \\
\hline 2005 & 292 & 3,20 & 8.200 .000 & $37.530,0$ & 1,00 & 0,12 & 0,94 & 0,78 & 0,79 & 0,58 & 13,23 & 4,98 & 4,45 & 4,0 \\
\hline 2006 & 292 & 0,02 & 8.300 .000 & $41.360,4$ & 1,03 & 0,12 & 1,09 & 0,79 & 0,79 & 0,59 & 24,05 & 12,21 & 9,51 & 3,9 \\
\hline 2003 & 293 & 0,15 & 4.600 .000 & $17.684,4$ & 0,95 & 0,12 & 0,75 & 0,79 & 0,79 & 0,57 & 12,27 & 7,43 & 6,64 & 3,7 \\
\hline 2004 & 293 & 4,20 & 4.600 .000 & $15.996,4$ & 0,66 & 0,08 & 0,37 & 0,79 & 0,79 & 0,58 & 21,00 & 21,96 & 17,56 & 3,8 \\
\hline 2005 & 293 & $-3,06$ & 4.000 .000 & $13.948,2$ & 0,78 & 0,10 & 0,75 & 0,78 & 0,79 & 0,58 & 1,80 & 0,66 & 0,67 & 4,0 \\
\hline 2006 & 293 & $-0,68$ & 4.800 .000 & $14.315,6$ & 0,71 & 0,11 & 1,04 & 0,79 & 0,79 & 0,59 & 5,08 & 1,20 & 1,19 & 3,9 \\
\hline 2003 & 342 & 0,59 & 4.500 .000 & $28.468,4$ & 0,55 & 0,06 & 1,05 & 0,79 & 0,79 & 0,57 & 9,78 & 17,27 & 15,68 & 3,7 \\
\hline 2004 & 342 & 0,49 & 4.200 .000 & $18.698,1$ & 0,29 & 0,07 & 1,98 & 0,79 & 0,79 & 0,58 & 3,96 & 6,62 & 6,40 & 3,8 \\
\hline 2005 & 342 & 0,05 & 4.400 .000 & $31.301,2$ & 0,31 & 0,05 & 0,95 & 0,78 & 0,79 & 0,58 & 2,55 & 6,10 & 5,97 & 4,0 \\
\hline 2006 & 342 & $-0,12$ & 361.329 & $38.405,3$ & 0,42 & 0,00 & 1,01 & 0,79 & 0,79 & 0,59 & 1,75 & 6,89 & 6,78 & 3,9 \\
\hline
\end{tabular}


Juan Carlos Trujillo Lora • Wilman Iglesias Pinedo

\begin{tabular}{|c|c|c|c|c|c|c|c|c|c|c|c|c|c|c|}
\hline Años & CIIU & Cl & SPI & RKP & koratio & CLU & ICL & ILE & idh & gini & TAE & TPI & TE & Corrup \\
\hline 2003 & 343 & 0,17 & 7.200 .000 & $36.969,4$ & 1,10 & 0,11 & 1,03 & 0,79 & 0,79 & 0,57 & 17,12 & 25,60 & 21,39 & 3,7 \\
\hline 2004 & 343 & 0,18 & 7.000 .000 & $33.370,7$ & 0,91 & 0,08 & 1,18 & 0,79 & 0,79 & 0,58 & 12,40 & 11,40 & 10,11 & 3,8 \\
\hline 2005 & 343 & 1,29 & 6.900 .000 & $26.854,9$ & 0,76 & 0,09 & 0,76 & 0,78 & 0,79 & 0,58 & 14,32 & 16,16 & 13,99 & 4,0 \\
\hline 2006 & 343 & 0,19 & 7.100 .000 & $27.813,0$ & 0,81 & 0,09 & 0,96 & 0,79 & 0,79 & 0,59 & 12,36 & 13,21 & 11,70 & 3,9 \\
\hline 2003 & 359 & 0,93 & 5.500 .000 & $16.354,7$ & 0,56 & 0,06 & 0,89 & 0,79 & 0,79 & 0,57 & 8,55 & 36,56 & 33,52 & 3,7 \\
\hline 2004 & 359 & 1,05 & 5.800 .000 & $20.375,5$ & 0,39 & 0,04 & 0,75 & 0,79 & 0,79 & 0,58 & 2,00 & 7,86 & 7,72 & 3,8 \\
\hline 2005 & 359 & $-2,68$ & 5.500 .000 & $12.400,7$ & 0,39 & 0,08 & 0,96 & 0,78 & 0,79 & 0,58 & 0,18 & 11,23 & 11,21 & 4,0 \\
\hline 2006 & 359 & 0,54 & 5.400 .000 & $10.515,6$ & 0,29 & 0,07 & 0,97 & 0,79 & 0,79 & 0,59 & 0,65 & 1,98 & 1,97 & 3,9 \\
\hline
\end{tabular}

Fuente: elaboración propia 